\title{
Flexible Polydimethylsiloxane Foams Decorated with Multiwalled Carbon Nanotubes Enable Unprecedented Detection of Ultralow Strain and Pressure Coupled with a Large Working Range
}

Rossella Iglio, ${ }^{\dagger}$ Stefano Mariani, ${ }^{\dagger}$ Valentina Robbiano, ${ }^{\dagger}$ Lucanos Strambini, $^{\ddagger}$ and Giuseppe Barillaro ${ }^{*}, \dagger, \ddagger 0$

${ }^{\dagger}$ Dipartimento di Ingegneria dell’Informazione, Università di Pisa, via G. Caruso 16, 56122, Pisa, Italy

${ }^{\ddagger}$ Istituto di Elettronica e di Ingegneria dell'Informazione e delle Telecomunicazioni, Consiglio Nazionale delle Ricerche, via G. Caruso 16, 56122, Pisa, Italy

\section{Supporting Information}

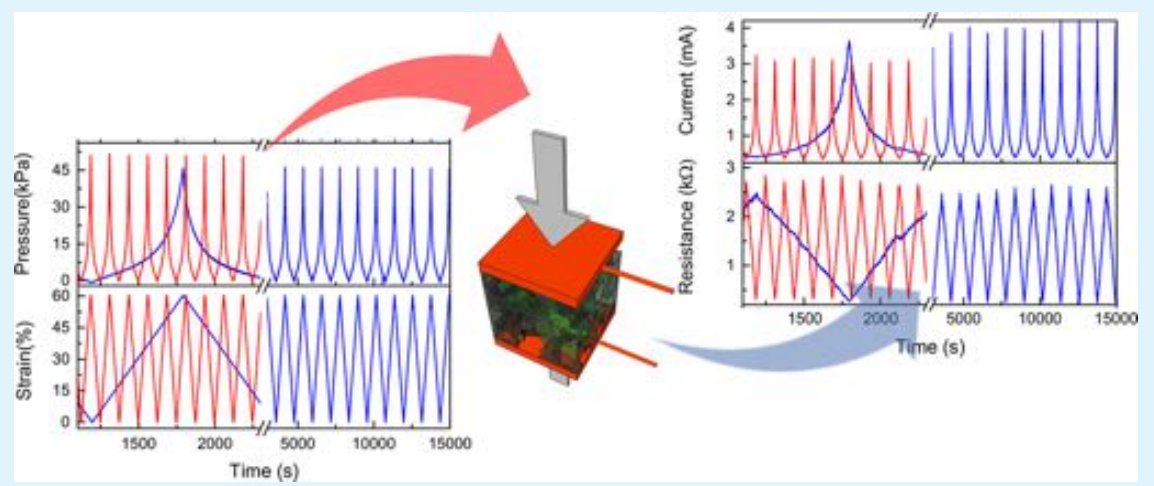

ABSTRACT: Low-cost piezoresistive strain/pressure sensors with large working range, at the same time able to reliably detect ultralow strain $(\leq 0.1 \%)$ and pressure $(\leq 1 \mathrm{~Pa})$, are one of the challenges that have still to be overcome for flexible piezoresistive materials toward personalized health-monitoring applications. In this work, we report on unprecedented, simultaneous detection of ultrasmall strain $(0.1 \%$, i.e., $10 \mu \mathrm{m}$ displacement over $10 \mathrm{~mm})$ and subtle pressure $(20 \mathrm{~Pa}$, i.e., a force of only $2 \mathrm{mN}$ over an area of $1 \mathrm{~cm}^{2}$ ) in compression mode, coupled with a large working range (i.e., up to $60 \%$ for strain $-6 \mathrm{~mm}$ in displacement-and $50 \mathrm{kPa}$ for pressure) using piezoresistive, flexible three-dimensional (3D) macroporous polydimethylsiloxane (pPDMS) foams decorated with pristine multiwalled carbon nanotubes (CNTs). pPDMS/CNT foams with pore size up to $500 \mu \mathrm{m}$ (i.e., twice the size of those of commonly used foams, at least) and porosity of $77 \%$, decorated with a nanostructured surface network of CNTs at densities ranging from 7.5 to $37 \mathrm{mg} / \mathrm{cm}^{3}$ are prepared using a low-cost and scalable process, through replica molding of sacrificial sugar templates and subsequent drop-casting of CNT ink. A thorough characterization shows that piezoresistive properties of the foams can be finely tuned by controlling the CNT density and reach an optimum at a CNT density of $25 \mathrm{mg} /$ $\mathrm{cm}^{3}$, for which a maximum change of the material resistivity (e.g., $\rho_{0} / \rho_{50}=4$ at $50 \%$ strain) is achieved under compression. Further static and dynamic characterization of the pPDMS/CNT foams with $25 \mathrm{mg} / \mathrm{cm}^{3}$ of CNTs highlights that detection limits for strain and pressure are $0.03 \%(3 \mu \mathrm{m}$ displacement over $10 \mathrm{~mm})$ and $6 \mathrm{~Pa}\left(0.6 \mathrm{mN}\right.$ over an area of $\left.1 \mathrm{~cm}^{2}\right)$, respectively; moreover, good stability and limited hysteresis are apparent by cycling the foams with 255 compression-release cycles over the strain range of $0-60 \%$, at different strain rates up to $10 \mathrm{~mm} / \mathrm{min}$. Our results on piezoresistive, flexible pPDMS/CNT foams pave the way toward breakthrough applications for personalized health care, though not limited to these, which have not been fully addressed to date with flexible strain/stress sensors.

KEYWORDS: porous PDMS foams, carbon nanotubes, piezoresistivity, strain sensors, pressure sensors, flexible sensors, ultralow strain/pressure detection, large working range

\section{INTRODUCTION}

Wearable sensors with high flexibility, durability, and excellent sensitivity, coupled with low power consumption, biocompatibility, and light weight, to be either attached to clothing or directly mounted on the skin, have attracted tremendous attention for real-time monitoring of physical, chemical, biological signals/stimuli from/to the human body. ${ }^{1-3}$
Over the last years, a number of piezoresistive, piezocapacitive, and piezoelectric wearable strain ${ }^{2}$ and pressure ${ }^{3}$ sensors for health care applications has been reported using conductive, rigid nanomaterials used as sensing elements, coupled with

Received: February 7, 2018

Accepted: April 3, 2018

Published: April 3, 2018 
insulating, stretchable polymers used as flexible supports. This approach allows outstanding electrical and mechanical properties of metal- and carbon-based nanomaterials to be conjugated with high stretchability and durability of polymers, resulting in novel functional, composite materials that enable relatively large strain and pressure to be effectively addressed, yet ensuring high sensitivity at small strain and pressure values.

Among these, piezoresistive flexible sensors have been prepared with low-dimensional carbons (e.g., carbon blacks, ${ }^{4,5}$ carbon nanotubes (CNTs), ${ }^{6,7}$ and graphene ${ }^{8,9}$ ), nanofibers, ${ }^{10,11}$ and nanoparticles, ${ }^{12,13}$ using silicone-based elastomers (e.g., polydimethylsiloxane (PDMS), ${ }^{4-13}$ ecoflex ${ }^{14,15}$ ) and rubbers ${ }^{16,17}$ as flexible supports. Different mechanisms, such as tunnelling, crack propagation, disconnection, and interlocking between nanomaterials, have been used to boost sensitivity to small strain or pressure. ${ }^{4-17}$ However, these sensors have been typically optimized for either strain or pressure measurements and do not usually allow both these signals to be measured with high resolution over a large working range. For instance, Jeong et al. $^{8}$ reported on a highly stretchable and sensitive strain sensor fabricated using fragmentized graphene on a PDMS slab, which was able to detect a minimum strain of $0.08 \%$ over a range of $50 \%$. Pang et al. ${ }^{10}$ developed a strain-gauge sensor based on nanoscale mechanical interlocking between Pt-coated polymer nanofibers sandwiched within thin PDMS supports, which was able to detect pressure (under compression) of only $5 \mathrm{~Pa}$ over a working range of $1.5 \mathrm{kPa}$ and strain as low as $0.1 \%$, up to $2 \%$.

More recently, piezoresistive strain/pressure flexible sensors exploiting three-dimensional (3D) polymer foams (mostly PDMS) coated with conductive nanomaterials have attracted increased attention ${ }^{18-26}$ due to superior deformability characteristics of porous polymers, which greatly exceed those of their solid (i.e., nonporous) counterparts, ${ }^{27}$ as well as better dispersion properties of carbon-based nanomaterials in the porous polymer, which significantly improves electrical conductivity of the nanocomposites. ${ }^{19}$ For instance, Yao et al. $^{18}$ reported graphene-wrapped polyurethane (PU) sponges coated with fractured graphene oxide nanosheets through solution dipping, which were able to reliably sense a minimum pressure (under compression) of $9 \mathrm{~Pa}$, up to $2 \mathrm{kPa}$. Song et $\mathrm{al}^{25}$ reported on compressible PDMS sponges coated with CNTs, prepared by drop-casting of CNT ink into sacrificial sugar cubes, which were able to sense pressure and strain in the range of $26 \mathrm{~Pa}$ to $150 \mathrm{kPa}$ and $10-60 \%$, respectively. Lv et al. ${ }^{23}$ reported on a pressure sensor based on a fully air-bubbled ultralight graphene block fabricated through a sparkling strategy, which exhibited reliable detection of ultralow to high strain and pressure, namely, from 0.1 to $90 \%$ and from $0.6 \mathrm{~Pa}$ $\left(0.03 \mathrm{mN}\right.$ over an area of $\left.0.49 \mathrm{~cm}^{2}\right)$ to about $1 \mathrm{kPa}$, respectively.

From the analysis of the state-of-the-art literature on both bulk and porous flexible strain and pressure sensors, it is apparent that, in spite of the different approaches that have been used for their fabrication, it is still a great challenge to achieve piezoresistive sensors with simultaneous ultralow limits of detection and large working ranges both for pressure and strain, which are able to address subtle $(1 \mathrm{~Pa}$ to $1 \mathrm{kPa})$ to medium (up to $100 \mathrm{kPa}$ ) pressures ${ }^{3}$ and ultrasmall $(\leq 0.1 \%)$ to large $(>50 \%)$ strains. $^{2}$

Here, we report on piezoresistive, flexible, three-dimensional (3D) macroporous PDMS (pPDMS) foams with pores of about $500 \mu \mathrm{m}$, twice the size of those of commonly used foams, decorated with a complex network of pristine multiwalled CNTs (density of $25 \mathrm{mg} / \mathrm{cm}^{3}$ ), enabling reliable and simultaneous detection of ultrasmall strain $(0.1 \%)$ and pressure $(20 \mathrm{~Pa})$ in compression mode, coupled with a large dynamic range (i.e., up to $60 \%$ for strain $-6 \mathrm{~mm}$ displacement-and 50 $\mathrm{kPa}$ for pressure).

\section{RESULTS AND DISCUSSION}

Preparation and Morphological Characterization of pPDMS Foams Decorated with CNTs. Preparation of pPDMS foams decorated with CNTs is sketched in Figure 1a. Commercial sugar cubes with grain sizes of either $500 \pm$ 300 or $125 \pm 75 \mu \mathrm{m}$ (grain range of $800-200$ and $200-50 \mu \mathrm{m}$, respectively) and side of about $1 \mathrm{~cm}$ are used as sacrificial templates (Figure 1a-1). The sugar templates are infiltrated with PDMS prepolymer (1:10 base:curing components by weight) through capillarity action in a vacuum chamber (residual pressure is in the millibar range) and then cured at $65{ }^{\circ} \mathrm{C}$ for $4 \mathrm{~h}$ to enable complete PDMS polymerization (Figure 1a-2). Dissolution of the sugar templates in deionized water at $60{ }^{\circ} \mathrm{C}$ for $1 \mathrm{~h}$ results in a porous PDMS foam with pore sizes comparable to those of the sugar grains of the template. $^{28}$ The as-prepared pPDMS foam is dried in ambient conditions (Figure 1a-3), and then, decoration with CNTs is carried out by drop-casting of a number of drops $(0.8 \mathrm{~mL}$ each) of a CNT ink on top of the pPDMS foam. The CNT ink consists of a dispersion of pristine CNTs in ethanol with concentration of $2 \mathrm{mg} / \mathrm{mL}$, and the number of drops is set according to the mass of CNTs to be loaded in the pPDMS foam. After drop-casting, the foam is abundantly rinsed in ethanol to remove an excess of CNTs that are not tightly adsorbed on the pPDMS surface. Evaporation of ethanol in ambient conditions results in a pPDMS foam with CNTs adsorbed on the pore surface via physical interaction, which is mostly triggered by $\mathrm{CH}-\pi$ interactions between the methyl groups of PDMS and the $\pi$-electron-rich surface of CNTs ${ }^{29}$ (Figure 1a-4). pPDMS foams with different CNT densities ranging from about 7 to $37 \mathrm{mg} / \mathrm{cm}^{3}$ (after rinsing) are prepared. Electrical contacts are eventually attained through an adhesive/flexible copper tape placed on two opposite sides of the pPDMS/CNT foam (Figure 1a-5) and used to measure current/resistance variation of the pPDMS/CNT foam upon compressive strain/stress (Figure 1a-6).

In Figure $1 \mathrm{~b}$ images of pPDMS foams with $500 \pm 300 \mu \mathrm{m}$ pores (larger pore foams) before (white) and after (black) CNT decoration (both full-size and cross-section) are shown. CNTs uniformly decorate the foam surfaces, as it is apparent from both full-size and cross-sectional images. In Figure 1c, scanning electron microscope (SEM) images of a pPDMS/ CNT foam with $500 \pm 300 \mu \mathrm{m}$ pores (at different magnifications) are reported. The porous nature of the foam is apparent, with pore sizes in agreement to the grain sizes of the sugar template (Figure 1c-1); a complex network of CNTs with uniform distribution and random orientation is visible on the pore surface of the pPDMS foam (Figure 1c-2,3). Optical and SEM images of a pPDMS foam with $125 \pm 75 \mu \mathrm{m}$ pores (smaller pore foams) before and after CNT decoration are reported in the Supporting Information (Figure S1).

Figure $1 \mathrm{~d}$ and e summarizes porosity and contact angle values of pPDMS foams with both smaller and larger pores, measured before and after decoration with CNTs at different densities. Porosity (by definition void to total volume fraction) is evaluated by gravimetric measurements (three replicas for 
(a)
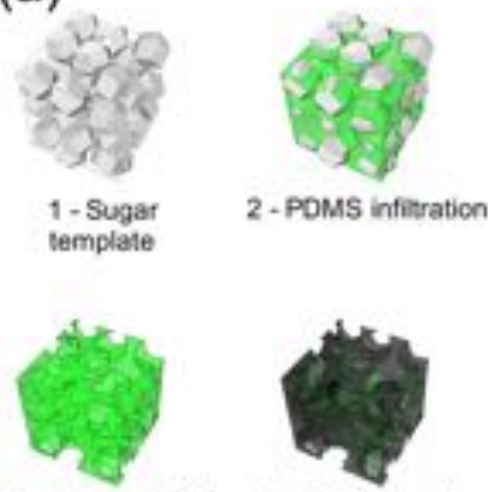

3 - Porous PDMS

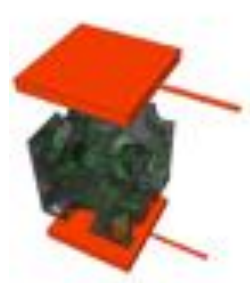

5 - Electrical contacts

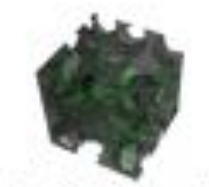

4 - CNT decoration

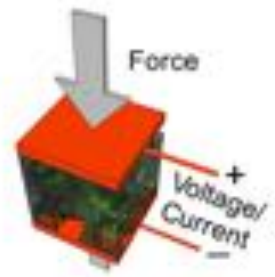

6-Actuation/Sensing (b)
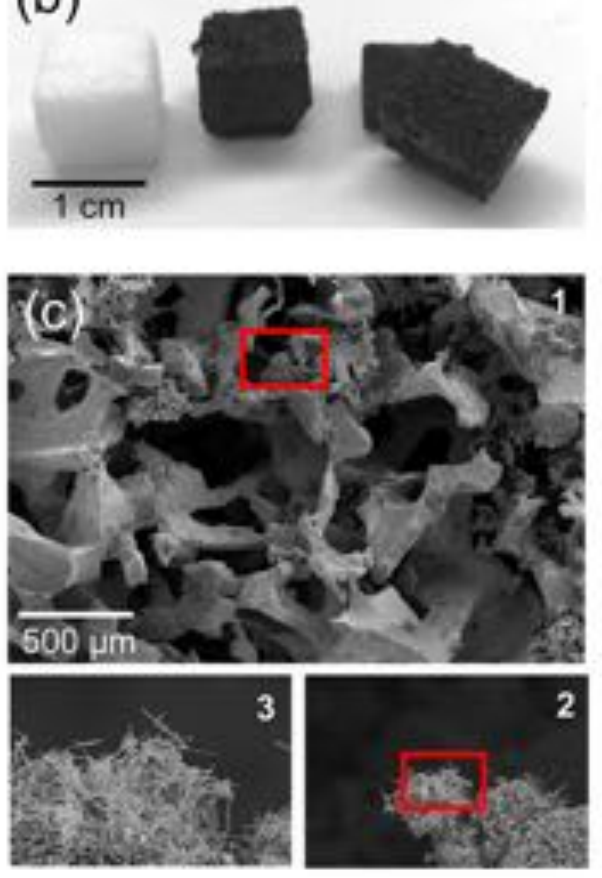

(d)

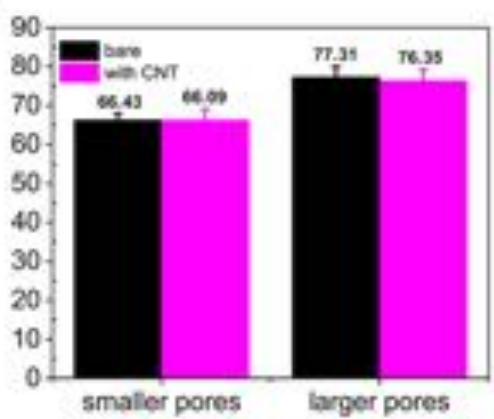

PPDMS

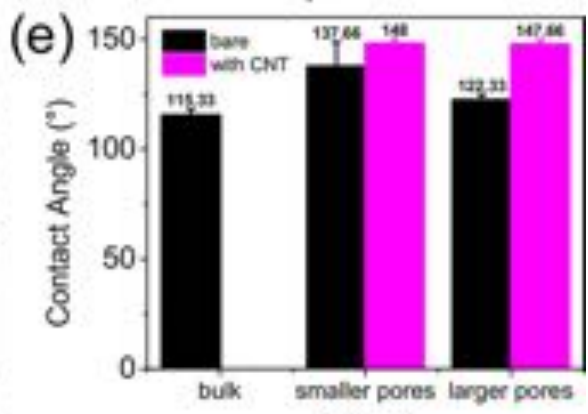

POMS

Figure 1. Preparation and morphological characterization of pPDMS/CNT foams. (a) Preparation steps of pPDMS foams and decoration with CNTs: (1) sketch of a sugar cube template; (2) PDMS prepolymer infiltration in the sugar cube in a vacuum chamber; (3) as-prepared pPDMS foam after PDMS thermal curing and dissolution of the sugar with warm water; (4) pPDMS foam decoration by drop casting of a number of controlled drops of a dispersion of pristine CNTs in ethanol; (5) formation of electrical contacts with copper tape on the two opposite sides of the foam; (6) sketch of the operation of the pPDMS/CNT foam as pressure/strain sensor through current/resistance monitoring. (b) Optical pictures of pPDMS foams with $500 \pm 300 \mu \mathrm{m}$ pores (larger pore foams) before (white) and after (black) CNT decoration (both full-size and cross-section). (c) Scanning electron microscope (SEM) images of the cross-section of a pPDMS/CNT foam with $500 \pm 300 \mu \mathrm{m}$ pores and CNT density of 25 $\mathrm{mg} / \mathrm{cm}^{3}$ at different magnifications: (1) 40×; (2) 1000×, and (3) 3500×. (d) Porosity of pPDMS foams with smaller and larger pores measured before (black) and after (magenta) decoration with different amounts of CNTs. (e) Static sessile contact angle measurements performed both on bulk PDMS, as well as on as-prepared (black) and CNT-decorated (magenta) pPDMS foams with both smaller and larger pores.

each CNT density), yielding a value of about 77 and $66 \%$ for pPDMS foams with larger and smaller pore sizes, respectively, regardless of the density of CNTs (Figure 1d). The porosity value of CNT-decorated foams is not significantly different from that of as-prepared foams (i.e., before CNT decoration), meaning that CNTs are mainly adsorbed on the surface of the pores and do not significantly change the overall pore volume of the pPDMS. This agrees well with SEM pictures in Figure $1 c$, where it is apparent that the CNTs arrange into a thin complex network conformably anchored to the pore surface.

Static sessile contact angle measurements, performed on bulk PDMS, as well as on as-prepared and CNT-decorated pPDMS foams using water $(5 \mu \mathrm{L})$, highlight an increase in the contact angle of porous PDMS with respect to bulk PDMS, namely, about $137^{\circ}$ and $122^{\circ}$ for smaller and larger pores, respectively, compared to $115^{\circ}$ of bulk PDMS (Figure 1e). We argue that the microstructured surface of pPDMS foams is responsible for the increased contact angle with respect to bulk PDMS, leading to a higher contact angle value as the pore size reduces. ${ }^{30,31}$ Decoration with CNTs further increases the contact angle of pPDMS foams to $148^{\circ}$, regardless of both average pore size and CNT density. We argue that the nanostructured CNT network formed on the pPDMS surface dominates the hydrophobicity of pPDMS/CNT foams, thus setting the contact angle of CNTdecorated foams to similar values in spite of the different CNT densities and pore sizes.
Mechanical Characterization of pPDMS Foams Decorated with CNTs. Figures $2 \mathrm{a}$ and S1c show typical stressstrain curves, with and without CNTs, of pPDMS foams with larger and smaller pores, respectively, measured under uniaxial compression. The curves point out a mechanical nonlinear behavior with hysteresis, which is typical of porous elastomeric materials. Two linear regions are apparent below $10 \%$ and above $90 \%$ of the maximum strain value, with slopes identifying low and high strain compressive modulus, ${ }^{32}$ respectively. Bare pPDMS foams with larger pores show a larger strain range (about $0-80 \%$ ) with respect to that of foams with smaller pores (about $0-60 \%$ ), which is also retained after decoration with CNTs. This has pushed further investigations of mechanical and electrical properties of pPDMS foams, both as-prepared and CNT-decorated, to be carried out only on those with larger pores, so as to take advantage of the $20 \%$ increase in strain range for pressure/strain sensing applications.

Decoration of bare pPDMS foams with CNTs significantly impacts the mechanical properties of the composite material, as shown in Figure 2a for pPDMS foams both as-prepared and decorated with two different CNT densities, namely, 7.5 and 37 $\mathrm{mg} / \mathrm{cm}^{3}$. It is apparent that whereas the lowest CNT density does not produce any significant change in the mechanical behavior of the foam, the highest CNT density greatly affects the foam behavior both in terms of elastic modulus and residual energy. Figure $2 \mathrm{~b}$ and $\mathrm{c}$ summarizes elastic modulus changes and residual energy changes of pPDMS foams decorated with 

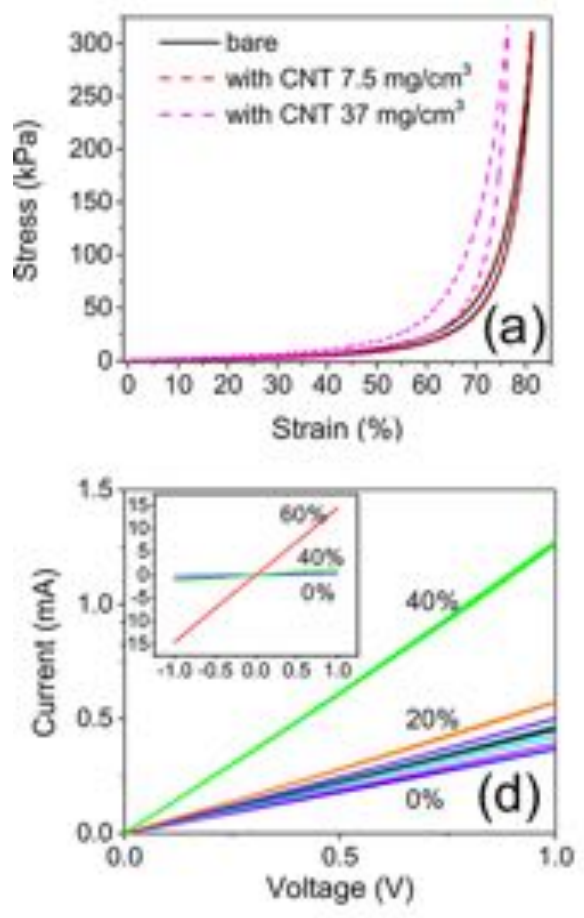
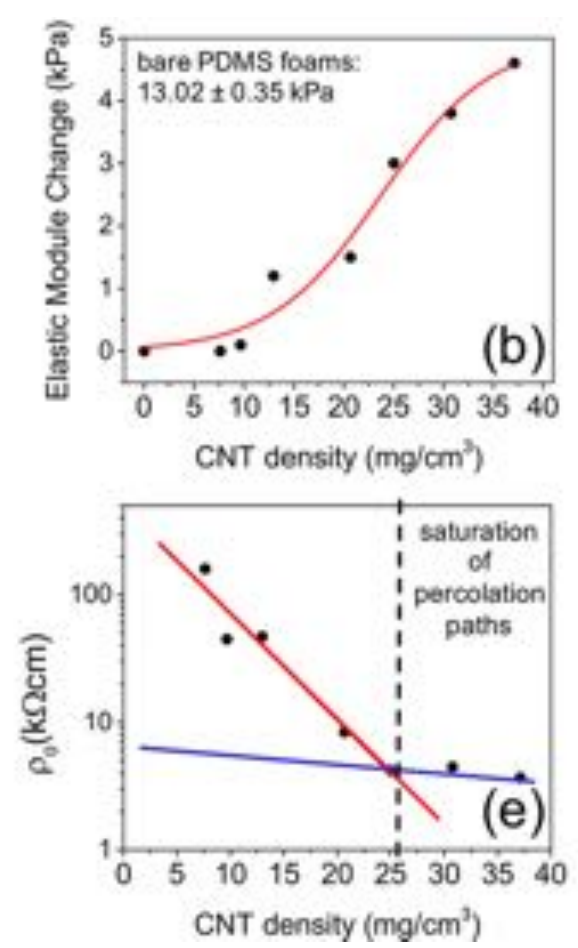
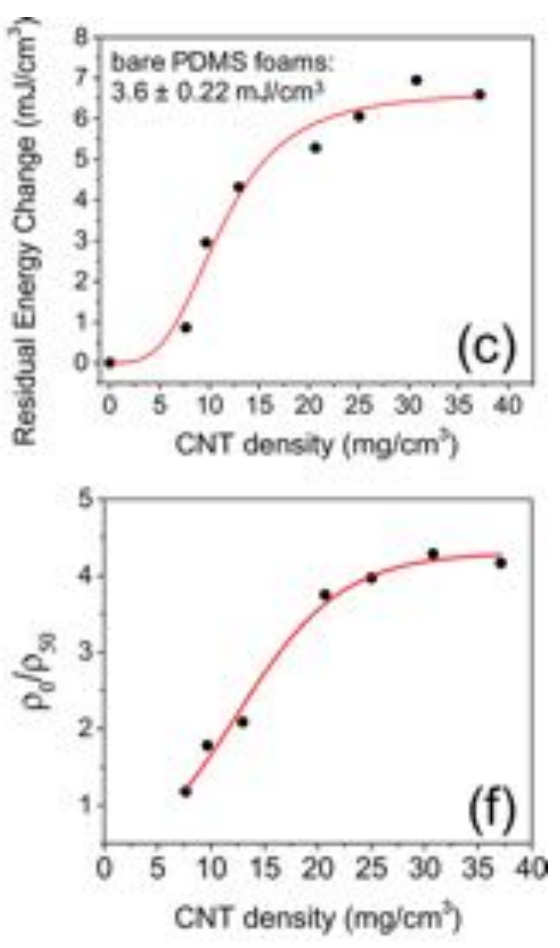

Figure 2. Mechanical and static piezoresistive characterization of pPDMS/CNT foams. (a) Stress-strain curves of pPDMS foams without (black line) and with CNT density of $7.5 \mathrm{mg} / \mathrm{cm}^{3}$ (dot red line) and $37 \mathrm{mg} / \mathrm{cm}^{3}$ (dot magenta line), measured under uniaxial compression (loading and unloading) at strain rate of $0.5 \mathrm{~mm} / \mathrm{min}$. (b) Elastic modulus change of pPDMS foams decorated with different densities of CNTs (black dots), with respect to bare PDMS foams. Experimental data are best-fitted by a logistic function (red line). (c) Residual energy change of pPDMS foams decorated with different densities of CNTs (black dots), with respect to bare PDMS foams. Experimental data are best-fitted by a logistic function (red line). (d) Current-voltage curves of pPDMS foams decorated with $25 \mathrm{mg} / \mathrm{cm}^{3}$ of CNTs in the range 0 to $+1 \mathrm{~V}$, recorded at different strain levels from 0 to $40 \%$; the inset shows the current-voltage curves over the range $\pm 1 \mathrm{~V}$ recorded at strain levels of 0,40 , and $60 \%$. (e) Resistivity at $0 \%$ strain, namely $\rho_{0}$, of pPDMS foams decorated with different CNT densities in the range of $7-37 \mathrm{mg} / \mathrm{cm}^{3}$ (black dots). Experimental data are bestfitted with a negative exponential function (red line) before saturation of the percolation paths occurring at $\mathrm{CNT}$ concentrations $>25 \mathrm{mg} / \mathrm{cm}^{3}$ (blue line). (f) Ratio of the resistivity of pPDMS/CNT foams at 0 and $50 \%$ strain, namely $\rho_{0} / \rho_{50}$, versus CNT density (black dots). Experimental data are best-fitted by a logistic function (red line).

different CNT densities, with respect to those of bare pPDMS foams. The elastic modulus of the foam (at strain <10\%) nonlinearly increases with the CNT density, with a maximum variation of $5 \mathrm{kPa}$ (i.e., $39 \%$ change) at $37 \mathrm{mg} / \mathrm{cm}^{3}$, with respect to that of bare PDMS foams (about $13 \mathrm{kPa}$ ) (Figure 2b). Best fitting of experimental data with a logistic function $\left(R^{2}=0.96\right)$ highlights that the elastic behavior of the pPDMS/CNT foam is marginally affected by the presence of CNTs at the lowest CNT densities; the change in the elastic modulus of the foam becomes more significant as the CNT density increases, with a saturation trend barely noticeable at the highest CNT densities. Interestingly, decoration of pPDMS with CNTs also affects the residual energy (i.e., the viscoelastic behavior) of the composite pPDMS/CNT foams, which increases with respect to that of bare pPDMS foams (about $3.6 \mathrm{~mJ} / \mathrm{cm}^{3}$ ) as the CNT density increases, with a maximum variation of about $7 \mathrm{~mJ} / \mathrm{cm}^{3}$ (i.e., about $200 \%$ change) at a CNT density of $37 \mathrm{mg} / \mathrm{cm}^{3}$. Best fitting of experimental data with a logistic function $\left(R^{2}=0.98\right)$ highlights that the residual energy of the pPDMS/CNT foams is significantly affected by the presence of CNTs already at the lowest CNT densities, with saturation at a CNT density of about $25 \mathrm{mg} / \mathrm{cm}^{3}$ (Figure $2 \mathrm{c}$ ).

We argue that changes of the mechanical properties (both elastic and viscoelastic) of the pPDMS/CNT foams with the density of CNTs loaded in the foam are mainly due to changes in the architecture of the CNT network formed on the pPDMS surface, which impacts the deformation of the pPDMS skeleton during both loading (i.e., compression) and unloading phases. On the one hand, at the lowest CNT densities, during the loading phase, most of the external energy is used to squeeze the pPDMS skeleton. As the CNT density increases, the network architecture becomes more complex and the energy required to modify the organization of the CNTs in the network increases. This agrees well with the observed increase of the elastic modulus of the foam with the CNT density. Further, during the loading phase, the CNT concentration per unit volume increases with strain, and a higher connection level among randomly oriented nanotubes establishes. At the end of this phase, a significant amount of the external energy used to compress the foam is stored in the CNT network, which is (the energy) fully released during the unloading phase to bring the foam back to its original state. On the other hand, release of the energy stored in the CNT network of compressed pPDMS/ CNT foams requires a rearrangement of the CNTs back to their initial positions, which occurs at a slower rate as the CNT density increases, due to the augmented interconnections among CNTs in the network after compression. This provides an apparent viscoelasticity contribution that increases with the density of CNTs loaded in the foam, which agrees well with the observed increase of the residual energy.

Characterization of pPDMS/CNT Foams for Ultralow Strain/Pressure Sensing. Static piezoresistive properties of the pPDMS/CNT foams loaded with different CNT densities 
are investigated by measuring $I-V$ curves at different strain levels from 0 to $60 \%$.

The pPDMS/CNT foams show a linear $I-V$ curve $\left(R^{2}>\right.$ 0.99 ), with a resistance value (i.e., inverse of the $I-V$ curve slope) that tremendously reduces as the strain level increases, for any tested CNT density (Figure 2d). The extent by which the resistance value is affected by the strain level significantly depends on the density of CNTs loaded in the foam. Figures $2 \mathrm{e}$ and $\mathrm{f}$ and S2a summarize the effect of the CNT density on the resistivity $\rho$ of pPDMS/CNT foams at two different strain levels, namely, 0 and $50 \%$. The resistivity value $\rho$ is obtained from the resistance value using the well-known relationship: $\rho=$ $(S / L) R$, where $L$ and $S$ are length and cross-sectional area of the foams at any given strain value. Given the small value of the Poisson ratio, namely $0.09 \pm 0.01$, of our pPDMS foams (measured at strain levels from $2.5 \%$ to $70 \%$ under uniaxial compression, Figure S3), a variation of the cross-sectional area $S$ of only $3.7 \%$ occurs under compression, with respect to its value at zero strain. This leads to a $3.7 \%$ error in resistivity by retaining the value of $S$ constant with strain. The use of the resistivity value for foam-to-foam comparison purposes allows possible artifacts affecting the resistance value of the foams to be effectively ruled out, such as, for instance, those related to slight variations in the pPDMS size among different foams.

The resistivity of the foam at zero strain (rest condition), namely $\rho_{0}$, exponentially decreases $\left(R^{2}=0.94\right.$, from best fitting of experimental data) with the CNT density, until it saturates around $4 \mathrm{k} \Omega \mathrm{cm}$ for CNT concentrations $>25 \mathrm{mg} / \mathrm{cm}^{3}$ (Figure 2e). The decrease of $\rho_{0}$ is explained in terms of increased number of percolation paths in the CNT network as the density of CNTs in the foam increases. Saturation of the percolation paths around CNT density of $25 \mathrm{mg} / \mathrm{cm}^{3}$ leads $\rho_{0}$ to level off beyond this CNT concentration value, in agreement with the literature. ${ }^{33}$ A similar trend in resistivity with the CNT density is also observed at strain level of $50 \%$, namely $\rho_{50}$, though resistivity values are dramatically smaller at strain of $50 \%$ than $0 \%$, at least at the highest CNT concentrations (Figure S2a). Consistently, saturation of $\rho_{50}$ around $1 \mathrm{k} \Omega \mathrm{cm}$ occurs at CNT densities $\geq 25 \mathrm{mg} / \mathrm{cm}^{3}$. We argue that, at the highest CNT densities, the number of interconnections among CNTs in the network is significantly increased at $50 \%$ strain, with respect to that at zero strain, which supports the remarkable decrease of the resistivity of the CNT network with strain. ${ }^{33}$ This agrees well with the outcomes on the mechanical characterization of pPDMS/CNT foams, which highlighted an increased CNT concentration per unit volume with strain.

Figure $2 \mathrm{f}$ shows the ratio $\rho_{0} / \rho_{50}$ versus CNT density, which clearly points out that the sensitivity of pPDMS/CNT foams to strain/stress increases with the CNT density, at least for CNT densities $\leq 25 \mathrm{mg} / \mathrm{cm}^{3}$. In fact, the $\rho_{0} / \rho_{50}$ ratio is about 1 at the smallest CNT concentration $\left(7.5 \mathrm{mg} / \mathrm{cm}^{3}\right)$ and increases to about 4 at the highest CNT concentrations $\left(\geq 25 \mathrm{mg} / \mathrm{cm}^{3}\right)$. This corroborates our hypothesis that changes in the material resistivity through rearrangement of the CNTs in the network upon compression of the foam play an important role as the CNT density increases. Notice that, the ratio $\rho_{0} / \rho_{50}$ is expected to be 1 for $50 \%$ strain if the material resistivity is retained constant with strain. The value of $\rho_{0} / \rho_{50}$ saturates to about 4 at CNT densities greater than $25 \mathrm{mg} / \mathrm{cm}^{3}$, which is consistent with the saturation of percolation paths pointed out both at zero and 50\% strain beyond this CNT concentration value.

Figure 3a shows typical relative resistance values versus strain levels of a pPDMS foam decorated with $25 \mathrm{mg} / \mathrm{cm}^{3}$ of CNTs.
The foam has a gauge factor $\mathrm{GF}=\frac{\mathrm{d} \Delta R / R_{0}}{\mathrm{~d} \Delta L / L_{0}}=5.6$ for strain $<2.5 \%$ and of 1.16 for strain from 2.5 to $60 \%$. The changes of the resistance are fully consistent with the aforementioned variation of the resistivity of the foam with strain, as apparent from Figure $3 \mathrm{~b}$ where relative resistivity versus strain values are shown over the whole working range. Figure $3 \mathrm{c}$ shows relative current versus pressure values (measured at bias of $1 \mathrm{~V}$ ), pointing out a high sensitivity of the foam current for both low and high pressure ranges, namely $S=\frac{\mathrm{d} \Delta I / I_{0}}{\mathrm{~d} P}=0.3 \mathrm{kPa}^{-1}$ for pressure $<1 \mathrm{kPa}$ and $0.9 \mathrm{kPa}^{-1}$ at pressures from 15 to $50 \mathrm{kPa}$. GF and $S$ values clearly highlight a very good sensitivity of the pPDMS/CNT foam to both strain and pressure, over the whole working range.

Further experiments are carried out to investigate piezoresistive properties of pPDMS foams decorated with $25 \mathrm{mg} / \mathrm{cm}^{3}$ of CNTs at small strain $(<3 \%)$ and pressure $(<500 \mathrm{~Pa})$ levels, by real-time measurement of the current flowing through the foam, at bias of $1 \mathrm{~V}$, upon application of repetitive displacement steps (both loading and unloading) of 10, 20, and $50 \mu \mathrm{m}$. Typical time-resolved curves showing raw data of current variations measured upon application of displacement steps of 10, 20, and $50 \mu \mathrm{m}$ are shown in Figure 3d. It is apparent that displacement steps of $10 \mu \mathrm{m}$, and greater, are reliably measured over the noise floor. Figure $3 \mathrm{e}$ shows current variation steadystate values (measured over 3 replicates) versus both displacement (strain) and pressure values. Notice that, error bars in Figure $3 e$ represent the current variation standard deviation values calculated taking into account both compression and release steps. The small value of the error bars, with respect to average values, is a clear indication that electrical hysteresis of the foams is negligible in this strain/pressure range, in agreement with negligible mechanical hysteresis of the pPDMS/CNT foams up to strain (pressure) of $15 \%(5 \mathrm{kPa})$ (Figure 2a). Moreover, a good consistency of the piezoresistive behavior of the pPDMS/CNT foam is apparent at small strain/ pressure values, in spite of the displacement step size (Figure 3e).

In order to provide a reliable estimation of the limit of detection (LOD) of pPDMS/CNT foams with CNT density of $25 \mathrm{mg} / \mathrm{cm}^{3}$, current variation steady-state values are grouped in terms of displacement step sizes, which allows a robust statistic on the current variation produced by a given displacement step to be achieved (Figure 3f). It is apparent that the foam reliably senses a displacement step of $10 \mu \mathrm{m}$ (i.e., a pressure of about 20 $\mathrm{Pa}$ ) with signal-to-noise ratio $S / N=13.6$, being $N=\sigma_{0}=0.28$ $\mu \mathrm{A}$ the noise floor, calculated as the standard deviation of the current at zero strain $\left(I_{0}\right)$. By best fitting the experimental data in Figure $3 \mathrm{f}$ with a linear regression $\left(R^{2}=0.98\right)$, we are able to calculate strain and pressure LOD values, that is the minimum displacement and pressure values that the foam can reliably sense over the noise region $3.3 \sigma_{0}$. The achieved LOD values are impressively low, resulting to be $3 \mu \mathrm{m}$ for displacement (i.e., strain $0.03 \%$ ) and $6 \mathrm{~Pa}$ for pressure, in spite of the large working range of the foams, namely $60 \%$ for strain (i.e., $6 \mathrm{~mm}$ in displacement) and $50 \mathrm{kPa}$ for pressure. We argue that the larger size of the pores of our foams, namely, $500 \mu \mathrm{m}$ in average, which is at least twice the size of those of foams commonly used for piezoresistive materials, ${ }^{18-26}$ plays a major role in the ultrasmall pressure/strain sensing when decorated with a superficial network of CNTs, at the same time enabling a larger working range. 

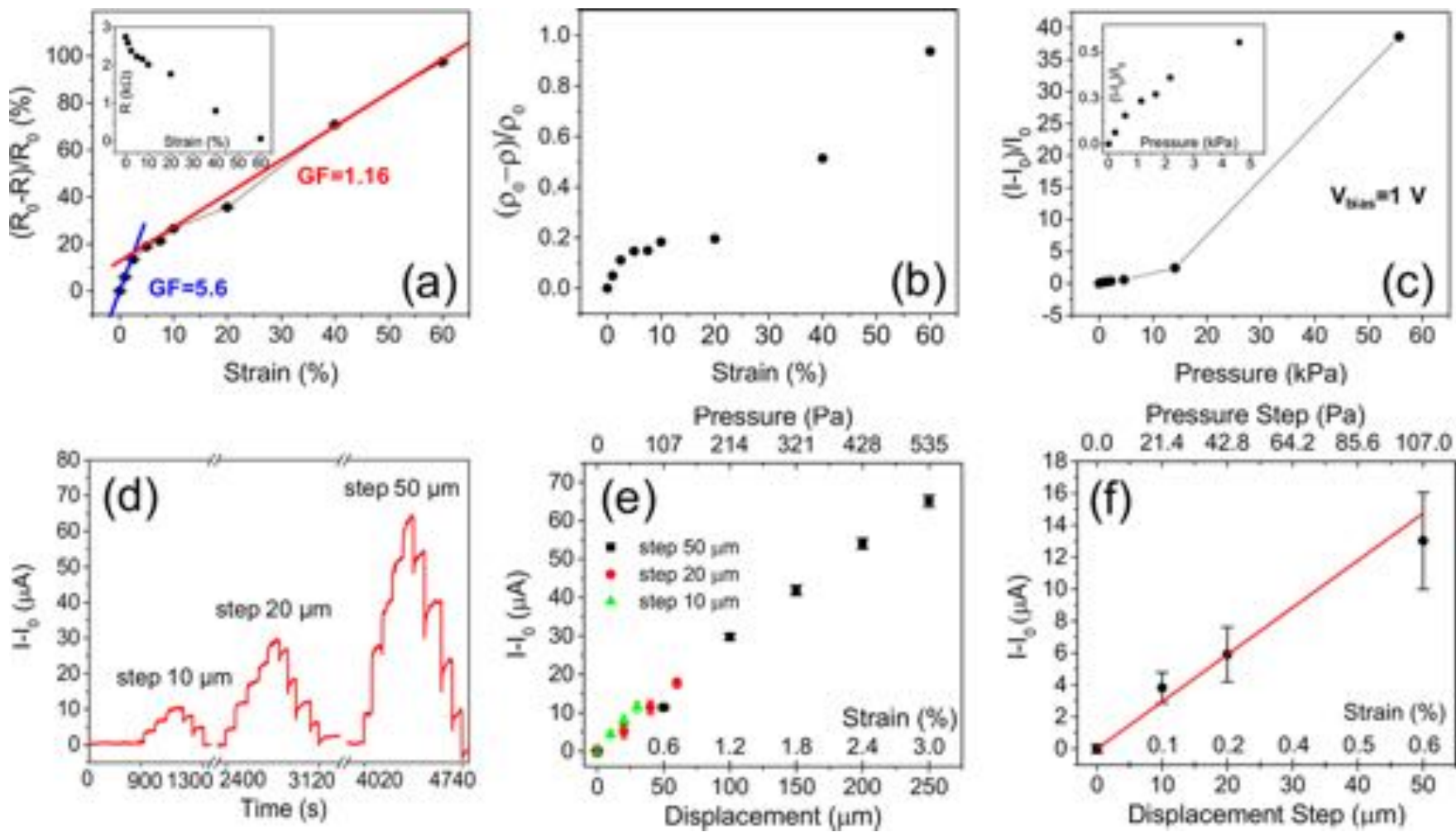

Figure 3. Piezoresitive properties of pPDMS/CNT foams for both large and ultralow strain/pressure regimes. (a) Normalized resistance variation at different compressive strain levels for a pPDMS foam decorated with $25 \mathrm{mg} / \mathrm{cm}^{3}$ of CNTs (black dots); the inset shows resistance values of the foam versus strain levels. The foam features a gauge factor (GF) of 5.6 in the range $0-2.5 \%$ (blue line) and of 1.16 from 2.5 to $60 \%$ (red line). (b) Normalized resistivity variation at different strain levels of the foam in a. (c) Relative current variation (at $1 \mathrm{~V}$ ) for different pressure values of the foam in a; the inset shows a magnification of the curve in the range $0-5 \mathrm{kPa}$. (d) Time-resolved current variation measured on a pPDMS foam with CNT density of $25 \mathrm{mg} / \mathrm{cm}^{3}$ subjected to loading/unloading steps of 10,20, and $50 \mu \mathrm{m}$. (e) Current variation versus displacement/strain and pressure measured on a pPDMS/CNT foam as in d upon application of repetitive displacement steps of 10, 20, and $50 \mu \mathrm{m}$ (data represent mean value and standard deviation of both loading and unloading steps over three replicates). (f) Current variation versus both displacement/strain and pressure step sizes of the pPDMS/CNT foam in e for strain up to $0.6 \%$ and pressure up to $100 \mathrm{~Pa}$ (data represent mean value and standard deviation of both loading and unloading steps over three replicates, black dots). Experimental data are best-fitted by a linear function (red line).

Dynamic Characterization of pPDMS/CNT Foams as Strain/Pressure Sensors. Dynamic piezoresistive properties of pPDMS/CNT foams with different CNT densities are investigated by simultaneously measuring both stress and current/resistance in real time under uniaxial compression (loading/unloading) at strain rates from 0.5 to $10 \mathrm{~mm} / \mathrm{min}$, in the range $0-60 \%$ and at biasing voltage of $1 \mathrm{~V}$.

Figure 4a shows time-resolved experimental raw data (10 full cycles) of (from top to bottom) current, resistance, and pressure acquired at strain rate of 0.5 (blue trace) and $5 \mathrm{~mm}$ / min (red trace) on a pPDMS/CNT foam loaded with $25 \mathrm{mg} /$ $\mathrm{cm}^{3}$ of CNTs. It is immediately apparent that a good correlation exists between resistance and strain and between current and pressure. In fact, as the strain increases (decreases) linearly over the range $0-60 \%$ (Figure $4 \mathrm{a}$, bottom), the resistance of the foam linearly decreases (increases) between $2.6 \mathrm{k} \Omega$ and $200 \Omega$, which is in good agreement with the piezoresistive behavior of the foam obtained from static measurements. On the other hand, the pressure nonlinearly increases (decreases) between 0 and $48 \mathrm{kPa}$ with the strain, in agreement to the stress-strain curve of the pPDMS/CNT foam (Figure S2c). Remarkably, the current flowing through the foam increases (decreases) with the same nonlinear behavior observed for pressure.

Current and resistance changes closely reproduce pressure and strain changes, with no significant delay and relatively small electrical hysteresis (maximum value $<10 \%$ at strain around $50 \%)$, meaning that the viscoelastic behavior of pPDMS/CNT foams does not significantly affect the piezoresistive performance of the foams at these strain rates. A good reproducibility of both current/resistance and pressure signals versus time is apparent over 20 continuous strain cycles, regardless of the strain rate value. This further confirms that pPDMS/CNT foams are both stable and reversible from electrical and mechanical points of view (Figure S2c). Further experiments carried out at a strain rate of $10 \mathrm{~mm} / \mathrm{min}$ for different strain ranges, namely about 15,30 , and $60 \%$, consistently confirm the high reproducibility and high sensitivity of piezoresistive properties of pPDMS/CNT foams (Figures S2d and S4).

The reliability of pPDMS/CNT foams is eventually investigated through application of 255 uniaxial compression/ release cycles over the strain range $0-60 \%$ at $10 \mathrm{~mm} / \mathrm{min}$ and measurement of current, resistance, and pressure versus time. Figure $4 \mathrm{~b}$ shows time-resolved values of current, resistance, pressure, and strain recorded on a pPDS/CNT foam with 25 $\mathrm{mg} / \mathrm{cm}^{3}$ of CNTs, biased at $1 \mathrm{~V}$. In particular, the full 255 cycles are reported in the left-hand side of the figure, whereas in the right-hand side of the figure a detail of 20 cycles acquired after $20 \mathrm{~min}$ (region 1) since the measurement began, superimposed onto 20 cycles acquired after $4 \mathrm{~h}$ (region 2 ) is shown. It is apparent that the piezoresistive properties of pPDMS/CNT foams are consistently and reliably retained after 

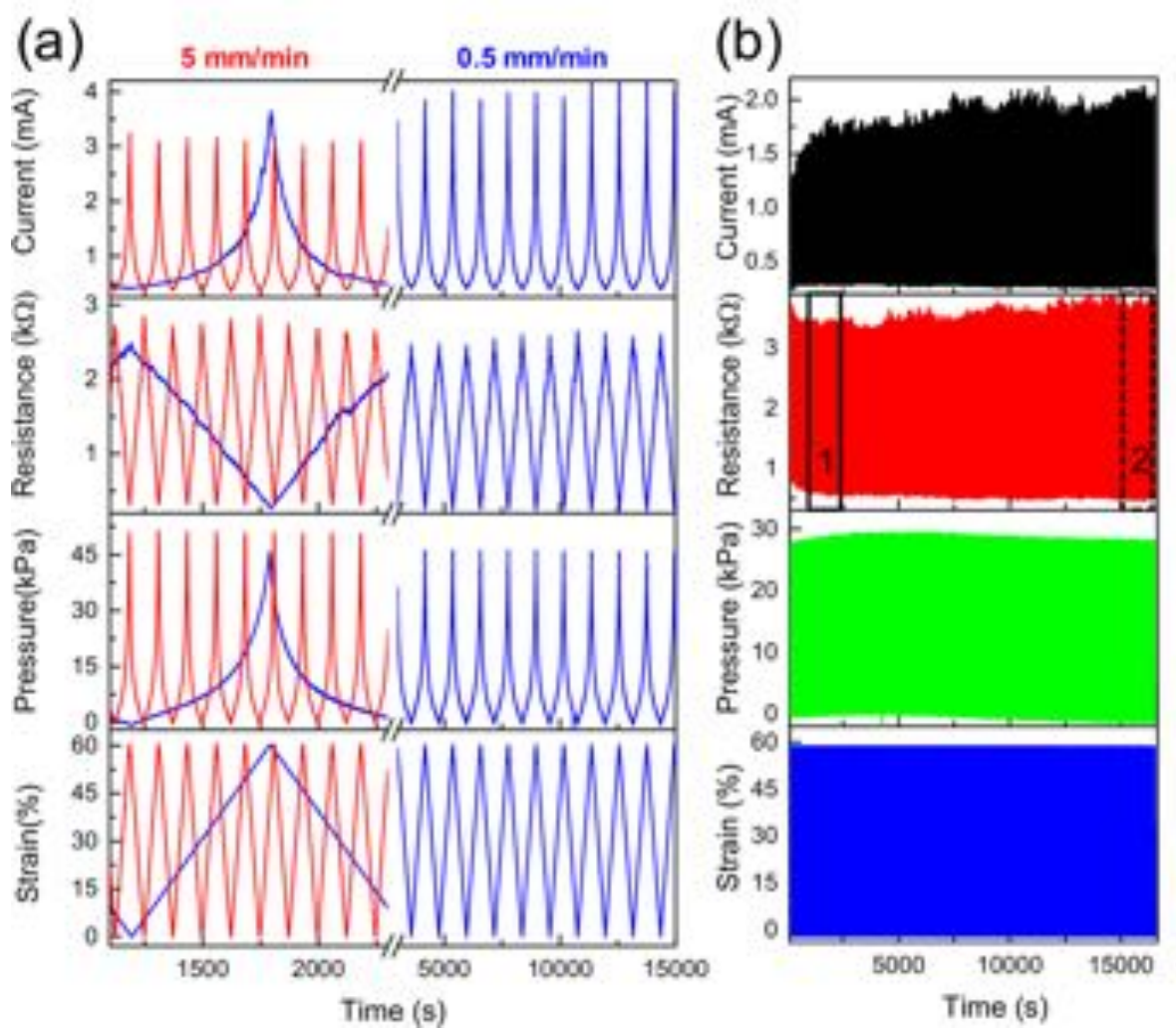

Time (s)

$15200 \quad 15400 \quad 15600 \quad 15600 \quad 16000 \quad 16200 \quad 16400$

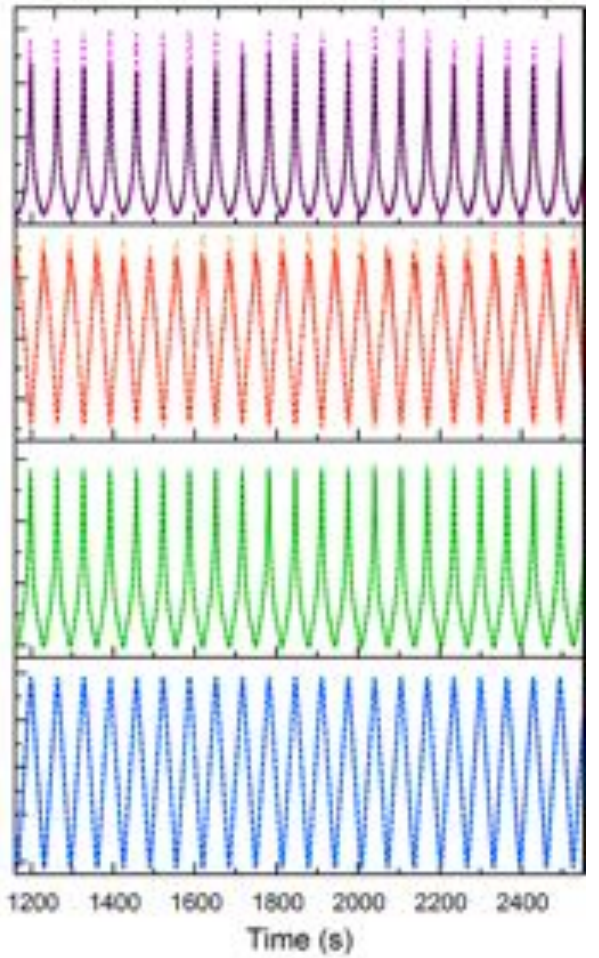

Figure 4. Dynamic piezoresistive properties of pPDMS/CNT foams at strain rates from 0.5 to $10 \mathrm{~mm} / \mathrm{min}$ in the range $0-60 \%$. (a) Time-resolved experimental data (10 full cycles) of current, resistance, and pressure acquired at strain rate of 0.5 (blue trace) and $5 \mathrm{~mm} / \mathrm{min}$ (red trace) on a pPDMS/CNT foam loaded with $25 \mathrm{mg} / \mathrm{cm}^{3}$ of CNTs, at bias of $1 \mathrm{~V}$. (b) Time-resolved experimental data (255 full cycles) of current, resistance, and pressure recorded at strain rate of $10 \mathrm{~mm} / \mathrm{min}$ acquired on a pPDMS/CNT foam with $25 \mathrm{mg} / \mathrm{cm}^{3}$ of CNTs, at bias of $1 \mathrm{~V}$ : (left) full $255 \mathrm{cycles}$, (right) detail of 20 cycles acquired after $20 \mathrm{~min}$ (region 1) since the measurement began, superimposed onto 20 cycles acquired after $4 \mathrm{~h}$ (region 2 ).

cycling the foams hundreds of times, thus confirming that CNTs are tightly anchored to the pPDMS surface and give rise to a randomly interconnected network that is reasonably robust and reliable both from electrical and mechanical points of view.

\section{CONCLUSIONS}

In this work, we gave the first-ever demonstration of reliable and simultaneous detection of ultrasmall strain (0.1\%) and subtle pressure $(20 \mathrm{~Pa})$ coupled with a large working range (up to $60 \%$ for strain and $50 \mathrm{kPa}$ for pressure) using piezoresistive, flexible three-dimensional (3D) macroporous PDMS (pPDMS) foams with pores of $500 \mu \mathrm{m}$, decorated with pristine multiwalled CNTs. The limit of detection of our pPDMS/ CNT foams is $0.03 \%$ (i.e., $3 \mu \mathrm{m}$ in displacement over $10 \mathrm{~mm}$ ) for strain and $6 \mathrm{~Pa}$ for pressure. The preparation of the pPDMS/CNT foams is low-cost, robust, and scalable, being based on simple replica molding of sacrificial sugar templates and subsequent drop-casting of CNT ink drops. Although no significant CNT losses were appreciated for the pPDMS/CNT foams of this work, further engineering of the foams to avoid any possible direct skin contact with CNTs is desirable for health care applications.

Our results envisage a disrupting role for flexible, piezoresistive strain/pressure sensors with ultralow limit of detection and large working range in high-precision personalized health care.

\section{EXPERIMENTAL SECTION}

Materials. Common sugar cubes, PDMS (polydimethylsiloxane), Sylgard 184 base (component A), and thermal curing agent (component B) are purchased from Dow Corning Corporation. Chemical vapor deposition (CVD) multiwalled carbon nanotubes (CNTs) (length 5-9 $\mu \mathrm{m}$, diameter $110-170 \mathrm{~nm}$, and purity $>90 \%$ ) and ethanol (99.9\%) are purchased from Sigma-Aldrich and used as received.

Preparation of Porous PDMS (pPDMS) Foams. pPDMS foams are prepared using sugar cubes with same dimension (about $1 \mathrm{~cm}$ in side) and different grain size (either $500 \pm 300 \mu \mathrm{m}$ from Dietor Vantaggio or $125 \pm 75 \mu \mathrm{m}$ from Persepolis) as sacrificial templates. PDMS components A and B are mixed in a ratio of 10:1 by weight (A:B) for 5 min in the Petri dish. A sugar cube is placed on top of the PDMS in the Petri dish, which is then put in a vacuum chamber (residual pressure a few $\mathrm{mBar}$ ) for $2 \mathrm{~h}$ with the twofold aim of ensuring full PDMS infiltration into the sugar template and removing air bubbles trapped in the PDMS itself. After infiltration with PDMS, the Petri dish is placed in a convection oven for $4 \mathrm{~h}$ at $65^{\circ} \mathrm{C}$ to ensure full PDMS polymerization through thermal curing. After PDMS curing, the Petri dish is then placed in a common freezer for $3 \mathrm{~min}$ to enable PDMS detachment from the sugar template scaffold. The sugar cube infiltrated with PDMS is removed from the bulk PDMS underneath using a razor blade, then it is put in a glass beaker with deionized water for $1 \mathrm{~h}$ at $60{ }^{\circ} \mathrm{C}$ to dissolve the sugar grains and obtain a porous PDMS foam. The pPDMS foam is eventually dried under a chemical hood for $1 \mathrm{~h}$ at room temperature.

Decoration of pPDMS Foams with CNTs. A CNT dispersion is prepared in a glass vial by adding $1 \mathrm{~mL}$ of ethanol for every $2 \mathrm{mg}$ of CNTs. The CNT dispersion is sonicated (frequency $40 \mathrm{kHz}$ ) for $5 \mathrm{~h}$ to obtain a homogeneous CNT dispersion. After sonication, several drops of the CNT dispersion with calibrated volume, namely $0.8 \mathrm{~mL}$ 
containing $1.65 \mathrm{mg}$ of CNTs, are drop-cast on top of the pPDMS foam. The number of drops is set according to the mass of CNTs to be loaded in the pPDMS foam. The PDMS foam is abundantly rinsed in ethanol (3 times) to remove CNTs that are not adsorbed on the PDMS surface, then air-dried under a chemical hood for $1 \mathrm{~h}$ at room temperature.

The mass of CNTs loaded in the pPDMS foam is obtained by weight difference of as-prepared and CNT-decorated foams, measured using an electronic precision balance (Kern ABS 220-4, weighing 220 $\mathrm{g}$, readout $0.1 \mathrm{mg}$, reproducibility $0.2 \mathrm{mg}$, and linearity $0.3 \mathrm{mg}$ ). The CNT density is calculated as the ratio between mass of loaded CNTs (mg) and volume of the foam $\left(\mathrm{cm}^{3}\right)$. The volume of the foam is evaluated measuring width, height, and thickness of each foam with a caliper.

Fabrication of Electrical Contacts on the pPDMS/CNT Foams. The electrical contacts of the pPDMS/CNT foams were attained through the use of a flexible copper tape (HB 720A Hi-Bond) placed on two opposite sides of the foam. The tape features a conductive acrylic flexible adhesive (with a uniform dispersion of conductive particles with thickness of $65 \mu \mathrm{m}$ and electrical resistivity of $10^{-1}-10^{-3} \Omega \mathrm{cm}$ ) that ensures reliable adhesion to the pPDMS/CNT foam even in absence of compression.

Morphological Characterization. The cross-sections of pPDMS foam decorated with CNTs are investigated using a scanning electron microscope (SEM, JEOL JSM-6390, Italy) at an accelerating voltage of $5 \mathrm{kV}$.

Porosity Measurements. Porosity of pPDMS foams, both before and after decoration with CNTs, is assessed by gravimetric measurements carried out either in air $\left(P_{\mathrm{a}}\right)$ through the use of eq 1 or in liquid $\left(P_{1}\right)$ through the use of eq 2 :

$$
\begin{aligned}
& P_{\mathrm{a}}=\frac{V_{\text {void }}}{V_{\mathrm{PDMS}}}=\frac{V_{\mathrm{PDMS}}-V_{\mathrm{pPDMS}}^{\mathrm{air}}}{V_{\mathrm{PDMS}}} \\
& P_{1}=\frac{V_{\text {void }}}{V_{\text {PDMS }}}=\frac{V_{\mathrm{pPDMS}}^{\mathrm{EtOH}}-V_{\mathrm{pPDMS}}^{\mathrm{air}}}{V_{\text {PDMS }}}
\end{aligned}
$$

In eq $1, V_{\text {PDMS }}$ is the volume of a nonporous (i.e., bulk) PDMS cube with same dimension of the porous foam and $V_{\text {void }}=V_{\text {PDMS }}-V_{\text {pPDMS }}$ is the pore volume of the pPDMS foam in air, being $V_{\mathrm{pPDMS}}=m_{\mathrm{pPDMS}} /$ $d_{\mathrm{PDMS}}$ the PDMS volume of the porous foam, $m_{\mathrm{pPDMS}}$, the weight of the porous foam, and $d_{\mathrm{PDMS}}$ the PDMS density. The mass of the pPDMS foam is $m_{\text {pPDMS }}$ measured through the use of an electronic precision balance (Kern ABS 220-4, weighing $220 \mathrm{~g}$, readout $0.1 \mathrm{mg}$, reproducibility $0.2 \mathrm{mg}$, and linearity $0.3 \mathrm{mg}$ ). The PDMS density $d_{\mathrm{PDMS}}=1.002 \mathrm{~g} / \mathrm{mL}$ is measured through preparation of four PDMS cubes with known size and calculation of the ratio between weight and volume of each cube.

In eq 2 , the pore volume is calculated as $V_{\text {void }}=\left(m_{\mathrm{pPDMS}}^{\mathrm{EtOH}}-\right.$ $\left.m_{\mathrm{pPDMS}}^{\text {air }}\right) / d_{\mathrm{EtOH}}$, where $m_{\mathrm{pPDMS}}^{\text {air }}$ and $m_{\mathrm{pPDMS}}^{\mathrm{EtOH}}$ are the weights of pPDMS foam in air and saturated with ethanol, respectively, and $d_{\mathrm{EtOH}}=0.789$ $\mathrm{g} / \mathrm{cm}^{3}$ is the ethanol density.

Contact Angle Measurements. Static sessile contact angle measurement tests were performed by placing a drop of deionized water with a volume of $5 \mu \mathrm{L}$ on the top side of the pPDMS foams both as-prepared and decorated with CNTs and measuring the angle between the solid/liquid interface (PDMS/water) through a computer graphics software (GIMP).

Mechanical Characterization. Mechanical characterization of pPDMS foams, both before and after CNT decoration, is carried out by cyclic uniaxial compressive tests using a Labview-controlled homemade setup (Figure S5) consisting of a $50 \mathrm{~N}$ load cell, used to measure the pressure to which pPDMS foams are subjected (Omega LCMFD, output $2 \mathrm{mV} / \mathrm{min}$, sensitivity $2.20 \mathrm{mV} / \mathrm{V}$, and accuracy 0.03 $\mathrm{N})$, a Source Meter unit to measure the output voltage of the load cell (Keithley SMU 2410), a translation motorized stage to impose the stress level on the pPDMS foams (Sigma Koki, stepping motor SGSP80-20ZF, travel per pulse $0.2 \mu \mathrm{m}$, max travel $20 \mathrm{~mm}$, max speed $2 \mathrm{~mm} / \mathrm{s}$ ), a stepping motor controller to finely control the translation stage (Sigma Koki, 2 axis stage controller SHOT-302GS, max number of steps 250 , min speed 1 pulse per second, max speed 500000 pulse per second).

Stress-strain curves of pPDMS both as-prepared and CNTdecorated foams are measured by imposing a linear strain (loading/ unloading, 3 full cycles) at a constant rate of $0.5 \mathrm{~mm} / \mathrm{min}$, with maximum travel of $10 \mathrm{~mm}$ and maximum force of $40 \mathrm{~N}$. From the stress-strain curves, both elastic modulus and residual energy of the foams are estimated as a function of the CNT density. Specifically, the elastic modulus is evaluated as the slope of the linear function best fitting experimental data in the strain range 0 to $10 \%$, within which the relationship between stress and strain is linear (Figure S6); the residual energy is calculated as the area under the stress-strain curve.

Electromechanical Characterization. Static piezoresistive properties of pPDMS/CNT foams are investigated in steady-state conditions through linear-sweep voltammetry for large strain levels and potentiostatic current measurements for small strain levels. The strain level/pressure value is set/measured using the homemade setup above-described for the mechanical characterization. An additional SMU (Keithley SMU 2410) is used to bias the pPDMS/CNT foams by applying the voltage through the copper tape contacts placed on opposite sides of the foams and measuring the current flowing through the foams. To ensure that any mechanical, and in turn electrical, transient due to pPDMS/CNT foam viscoelasticity is fully over, application of a given strain level is followed by a wait time of $5 \mathrm{~min}$ before electrical measurements are carried out. Experiments are repeated at least three times.

For large strain levels (namely, 0, 1, 2.5, 5, 7.5, 10, 20, 40, and 60\%), current-voltage curves of the pPDMS/CNT foams are recorded applying a voltage sweep ( 10 full cycles) in the range $\pm 1 \mathrm{~V}$ (step of $100 \mathrm{mV}$ ) to the foam and monitoring the resulting current for each voltage value. The resistance value $R$ versus strain level is extrapolated from current-voltage curves through best fitting with a linear regression. The resistivity value $\rho$ is obtained from the resistance value using the well-known relationship: $\rho=(S / L) R$, where $L$ and $S$ are length and cross-sectional area of the foams at any given strain value.

For small strain levels (namely, up to $3 \%$ ), repeated displacement

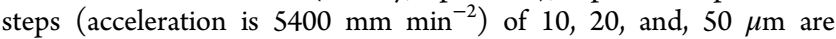
imposed to the foam and the current flowing through the foam is measured in real time at a bias of $1 \mathrm{~V}$.

Dynamic piezoresistive properties of pPDMS/CNT foams are investigated through cyclic uniaxial compression tests under constant biasing $(1 \mathrm{~V})$, using the same homemade setup above-described for the static piezoresistive characterization. A linear strain (loading/ unloading, minimum 20, maximum 255 full cycles) at different constant rates, namely $0.5,5$, and $10 \mathrm{~mm} / \mathrm{min}$, is applied to the foam. Different strain ranges, namely 15,30 , and $60 \%$, are investigated for any tested strain rate. For any tested condition, pressure, current $I$, and resistance $R=1 / I$ values of the pPDMS/CNT foams are measured over time every $0.35 \mathrm{~s}$.

\section{ASSOCIATED CONTENT}

\section{Supporting Information}

The Supporting Information is available free of charge on the ACS Publications website at DOI: 10.1021/acsami.8b02322.

Morphological and mechanical characterization of pPDMS foams with pores of $125 \pm 75 \mu \mathrm{m}$ (smaller pore foams) both as-prepared and after decoration with CNTs; piezoresistive and mechanical characteristics of pPDMS foams with pores of $500 \pm 300 \mu \mathrm{m}$ (larger pore foams) at different strain rates; dynamic piezoresistive characterization of pPDMS/CNT foams at $10 \mathrm{~mm} / \mathrm{min}$ at different strain ranges $(\mathrm{PDF})$

\section{AUTHOR INFORMATION}

\section{Corresponding Author}

*E-mail: g.barillaro@iet.unipi.it. 


\section{ORCID $\odot$}

Giuseppe Barillaro: 0000-0001-6197-4851

Notes

The authors declare no competing financial interest.

\section{ACKNOWLEDGMENTS}

This work was partially supported by the EU H2020 ETN SYNCHRONICS under grant agreement 643238.

\section{REFERENCES}

(1) Han, S. T.; Peng, H.; Sun, Q.; Venkatesh, S.; Chung, K. S.; Lau, S. C.; Zhou, Y.; Roy, V. A. L. An overview of the development of flexible sensors. Adv. Mater. 2017, 29, 1700375.

(2) Amjadi, M.; Kyung, K. U.; Park, I.; Sitti, M. Stretchable, skinmountable, and wearable strain sensors and their potential applications: a review. Adv. Funct. Mater. 2016, 26, 1678-1698.

(3) Zang, Y.; Zhang, F.; Di, C.; Zhu, D. Advances of flexible pressure sensors toward artificial intelligence and health care applications. Mater. Horiz. 2015, 2, 140-156.

(4) Lu, N.; Lu, C.; Yang, S.; Rogers, J. Highly sensitive skinmountable strain gauge based entirely on elastomers. Adv. Funct. Mater. 2012, 22, 4044-4050.

(5) Kong, J. H.; Jang, N. S.; Kim, S. H.; Kim, J. M. Simple and rapid micropatterning of conductive carbon composites and its application to elastic strain sensors. Carbon 2014, 77, 199-207.

(6) Yamada, T.; Hayamizu, Y.; Yamamoto, Y.; Yomogida, Y.; IzadiNajafabadi, A.; Futaba, D. N.; Hata, K. A stretchable carbon nanotube strain sensor for human-motion detection. Nat. Nanotechnol. 2011, 6, 296-301.

(7) Zhou, J.; Yu, H.; Xu, X.; Han, F.; Lubineau, G. Ultrasensitive, Stretchable strain sensors based on fragmented carbon nanotube papers. ACS Appl. Mater. Interfaces 2017, 9, 4835-4842.

(8) Jeong, J. R.; Park, H.; Jin, S. W.; Hong, S. Y.; Lee, S. S.; Ha, J. S. Highly stretchable and sensitive strain sensors using fragmentized graphene foam. Adv. Funct. Mater. 2015, 25, 4228-4236.

(9) Yan, C.; Wang, J.; Kang, W.; Cui, M.; Wang, X.; Foo, C. Y.; Chee, K. J.; Lee, P. S. Highly stretchable piezoresistive graphene-nanocellulose nanopaper for strain sensors. Adv. Mater. 2014, 26, 20222017.

(10) Pang, C.; Lee, G. Y.; Kim, T. I.; Kim, S. M.; Kim, H. N.; Ahn, S. H.; Suh, K. Y. A flexible and highly sensitive strain-gauge sensor using reversible interlocking of nanofibers. Nat. Mater. 2012, 11, 795-801.

(11) Chen, S.; Lou, Z.; Chen, D.; Jiang, K.; Shen, G. PolymerEnhanced highly stretchable conductive fiber strain sensor used for electronic data gloves. Advanced Materials Technologies 2016, 1, 1600136.

(12) Kang, D.; Pikhitsa, P. V.; Choi, Y. W.; Lee, C.; Shin, S. S.; Piao, L.; Park, B.; Suh, K. Y.; Kim, T.; Choi, M. Ultrasensitive mechanical crack-based sensor inspired by spider sensory system. Nature 2014, $516,222-226$.

(13) Lee, J.; Kim, S.; Lee, J.; Yang, D.; Park, B. C.; Ryu, S.; Park, I. Stretchable strain sensor based on metal nanoparticles thin film for human motion detection. Nanoscale 2014, 20, 1-8.

(14) Ryu, S.; Lee, P.; Chou, J. B.; Xu, R.; Zhao, R.; Hart, A. J.; Kim, S. G. Extremely elastic wearable carbon nanotube fiber strain sensor for monitoring of human motion. ACS Nano 2015, 9, 5929-5936.

(15) Muth, J. T.; Vogt, D. M.; Truby, R. L.; Mengüç, Y.; Kolesky, D. B.; Wood, R. J.; Lewis, J. A. Embedded 3D Printing of Strain Sensors within Highly Stretchable Elastomers. Adv. Mater. 2014, 26, 63076312 .

(16) Mannsfeld, S. C. B.; Tee, B. C. K.; Stoltenberg, R. M.; Chen, C. V. H. H.; Barman, S.; Muir, B. V. O.; Sokolov, A. N.; Reese, C.; Bao, Z. Highly sensitive flexible pressure sensors with microstructured rubber dielectric layers. Nat. Mater. 2010, 9, 859-864.

(17) Boland, C.; Khan, U.; Backes, C.; O’Neill, A.; Duane, S.; Shanker, R.; Liu, Y.; Jurewicz, I.; Dalton, A. B.; Coleman, N. J.; et al. Sensitive, high-strain, high-rate bodily motion sensors based on graphene-rubber composites. ACS Nano 2014, 8, 8819-8830.
(18) Yao, H. B.; Ge, J.; Wang, C. F.; Wang, X.; Hu, W.; Zheng, Z. J.; $\mathrm{Ni}$, Y.; Yu, S. H. A flexible and highly pressure-sensitive graphenepolyurethane sponge based on fractured microstructure design. Adv. Mater. 2013, 25, 6692-6698.

(19) Chen, M.; Zhang, L.; Duan, S.; Jing, S.; Jiang, H.; Li, C. Highly stretchable conductors integrated with a conductive carbon nanotube/ graphene network and 3D porous poly(dimethylsiloxane). Adv. Funct. Mater. 2014, 24, 7548-7556.

(20) Zhang, H.; Liu, N.; Shi, Y.; Yue, Y.; Wang, S.; Ma, Y.; Wen, L.; Li, L.; Long, F.; Zou, Z.; Gao, Y.; et al. Piezoresistive sensor with high elasticity based on 3D hybrid network of sponge@CNT@Ag NPs. ACS Appl. Mater. Interfaces 2016, 8, 22374-22381.

(21) Duan, S.; Yang, K.; Wang, Z.; Chen, M.; Zhang, L.; Zhang, H.; Li, C. Fabrication of Highly Stretchable Conductors Based on 3D Printed Porous Poly(dimethylsiloxane) and Conductive Carbon Nanotubes/Graphene Network. ACS Appl. Mater. Interfaces 2016, 8, 2187-2192.

(22) Li, J.; Zhao, S.; Zeng, X.; Huang, W.; Gong, Z.; Zhang, G.; Sun, R.; Wong, C. P. Highly stretchable and sensitive strain sensor based on facilely prepared three-dimensional graphene foam composite. ACS Appl. Mater. Interfaces 2016, 8, 18954-18961.

(23) Lv, L.; Zhang, P.; Xu, T.; Qu, L. Ultrasensitive pressure sensor based on an ultralight sparkling graphene block. ACS Appl. Mater. Interfaces 2017, 9, 22885-22892.

(24) Chen, H.; Su, Z.; Song, Y.; Cheng, X.; Chen, X.; Meng, B.; Song, Z.; Chen, D.; Zhang, H. Omnidirectional bending and pressure sensor based on stretchable CNT-PU sponge. Adv. Funct. Mater. 2017, 27, 1604434.

(25) Song, Y.; Chen, H.; Su, Z.; Chen, X.; Miao, L.; Zhang, J.; Cheng, X.; Zhang, H. Highly Compressible Integrated SupercapacitorPiezoresistance-Sensor System with CNT-PDMS Sponge for Health Monitoring. Small 2017, 13, 1702091.

(26) Wu, S.; Zhang, L.; Ladani, R. B.; Ravindran, A. R.; Mouritz, A. P.; Kinloch, A. J.; Wang, C. H. Novel Electrically Conductive Porous PDMS/Carbon Nanofiber Composites for Deformable Strain Sensors and Conductors. ACS Appl. Mater. Interfaces 2017, 9, 14207-14215.

(27) Park, J.; Wang, S.; Li, M.; Ahn, C.; Hyun, J. K.; Kim, D. S.; Kim, D. K.; Rogers, J. A.; Huang, Y.; Jeon, S. Three-dimensional nanonetworks for giant stretchability in dielectrics and conductors. Nat. Commun. 2012, 3, 1-8.

(28) Iglio, R.; Mariani, S.; Strambini, L. M.; Barillaro, G. Macroporous PDMS foam decorated with carbon nanotubes for conductometric pressure and strain sensors. IEEE Sensors 2017, 1.

(29) McNally, T.; Pötschke, P. Polymer-Carbon Nanotube Composites Preparation, Properties and Applications 2011, 741-742.

(30) Mittal, K. L. Contact angle, wettability and adhesion; CRC, Press, 2006; Vol. 4.

(31) Gu, H.; Wang, C.; Gong, S.; Mei, Y.; Li, H.; Ma, W. Investigation on contact angle measurement methods and wettability transition of porous surfaces. Surf. Coat. Technol. 2016, 292, 72-77.

(32) Cha, K. J.; Kim, D. S. A portable pressure pump for microfluidic lab-on-a-chip systems using a porous polydimethylsiloxane (PDMS) sponge. Biomed. Microdevices 2011, 13, 877.

(33) Alamusi; Hu, N.; Fukunaga, H.; Atobe, S.; Liu, Y.; Li, J. Piezoresistive strain sensors made from carbon nanotubes based polymer nanocomposites. Sensors 2011, 11, 10691-10723. 


\section{Supporting Information}

Flexible Polydimethylsiloxane Foams Decorated with Multiwalled Carbon Nanotubes Enable Unprecedented Detection of Ultralow Strain and Pressure Coupled With Large Working Range

Rossella Iglio $^{1}$, Stefano Mariani ${ }^{1}$, Valentina Robbiano ${ }^{1}$, Lucanos Strambini ${ }^{2}$, Giuseppe Barillaro ${ }^{1,2^{*}}$ ${ }^{1}$ Dipartimento di Ingegneria dell'Informazione, Università di Pisa, via G. Caruso 16, 56122, Pisa, Italy

${ }^{2}$ Istituto di Elettronica e di Ingegneria dell'Informazione e delle Telecomunicazioni, Consiglio Nazionale delle Ricerche, via G. Caruso 16, 56122, Pisa, Italy

*g.barillaro@iet.unipi.it

These Supporting Information provide additional details and data on: morphological and mechanical characterization of pPDMS foams with pores of $125 \pm 75 \mu \mathrm{m}$ (smaller pore foams) both as-prepared and after decoration with CNTs; piezoresistive and mechanical characteristics of pPDMS foams with pores of $500 \pm 300 \mu \mathrm{m}$ (larger pore foams) at different strain rates; dynamic piezoresistive characterization of pPDMS/CNT foams at $10 \mathrm{~mm} / \mathrm{min}$ at different strain ranges. 

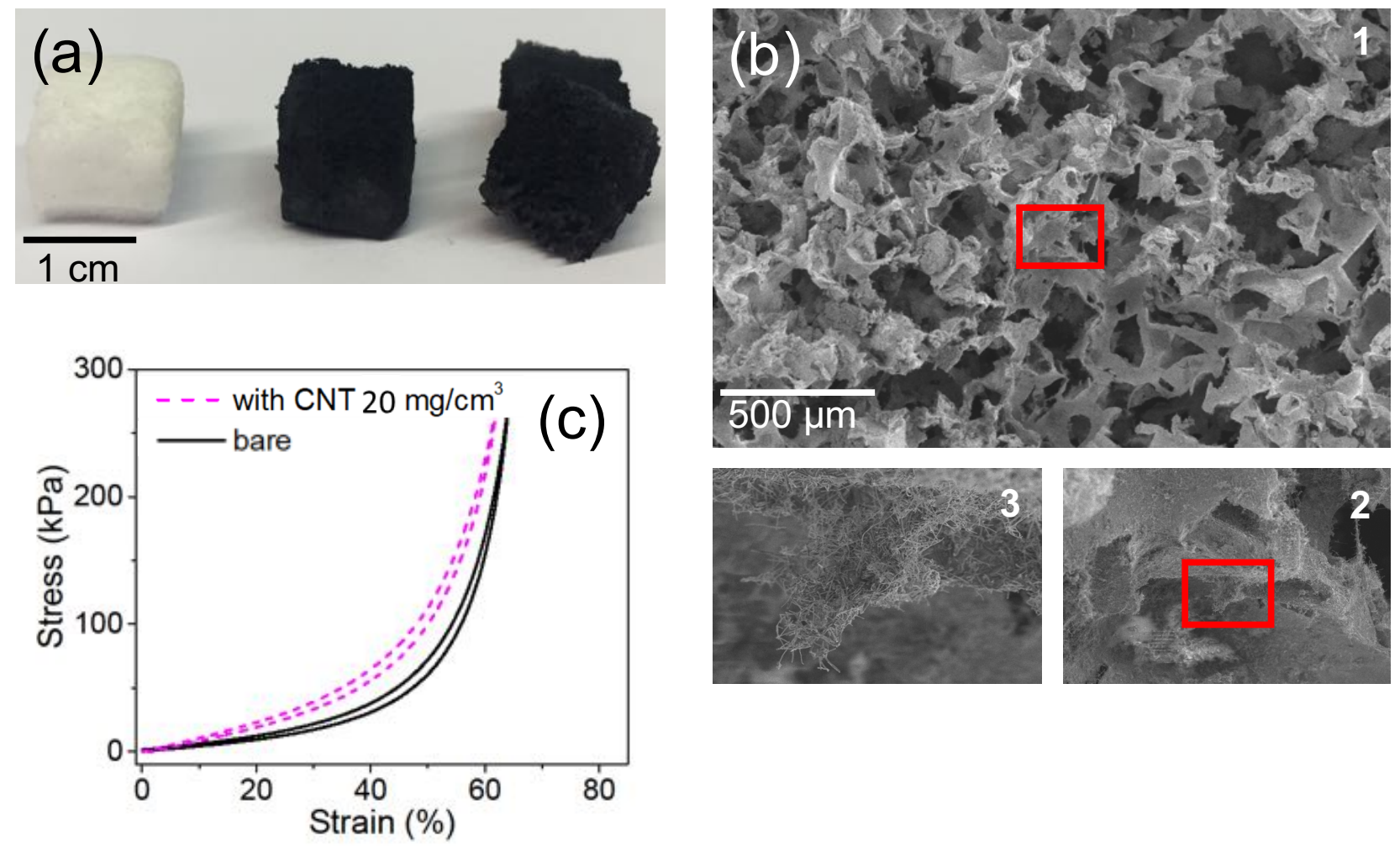

Figure S1 Morphological and mechanical characterization of pPDMS foams with $125 \pm 75 \mu \mathrm{m}$ (smaller pores). (a) Optical pictures of pPDMS foam before (white) and after (black) CNT decoration (full-size and cross-section). (b) SEM images of a pPDMS/CNT foam with $125 \pm 75 \mu \mathrm{m}$ pores decorated with CNTs at density of $20 \mathrm{mg} / \mathrm{cm}^{3}$ at different magnifications: (1) 40X; (2) 1000X; (3) 3500X. (c) Stress-strain curves of pPDMS foams without (black trace) and with CNTs at density of $20 \mathrm{mg} / \mathrm{cm}^{3}$ (magenta trace), measured under uniaxial compression loading and unloading at strain rate of $0.5 \mathrm{~mm} / \mathrm{min}$. 

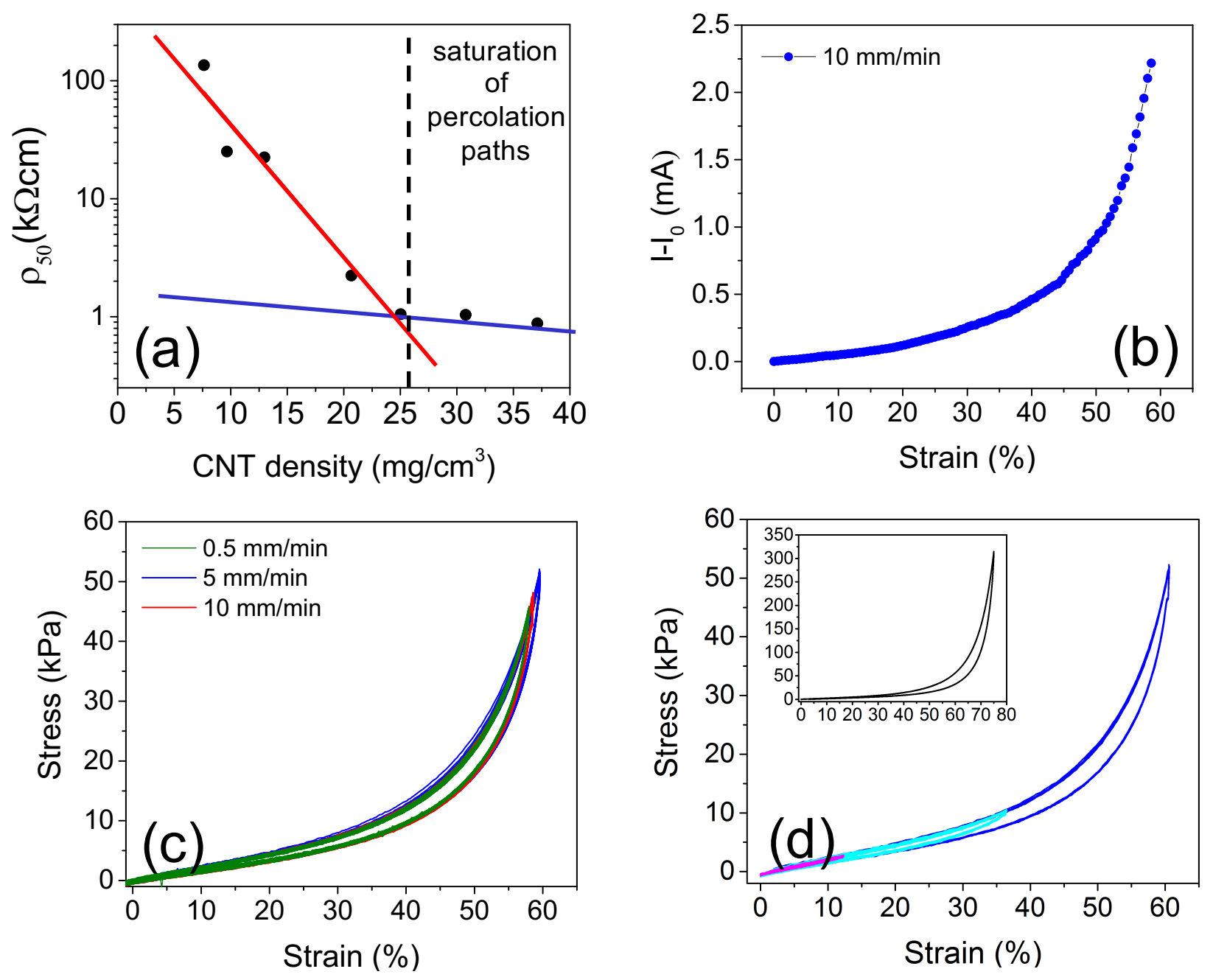

Figure S2 Static and dynamic piezoresistive and mechanical characterization of pPDMS/CNT

foams. (a) Resistivity at 50\% strain, namely $\rho_{50}$, of pPDMS foam decorated with different CNT densities in the range 7 to $37 \mathrm{mg} / \mathrm{cm}^{3}$ (black dots). Experimental data are best fitted with a negative exponential function (red line) before saturation of the percolation paths occurring at CNT concentrations $>25 \mathrm{mg} / \mathrm{cm}^{3}$ (blue line). (b) Current variation versus strain recorded during compressive strain tests at $10 \mathrm{~mm} / \mathrm{min}$, highlighting the linear relationship between current and strain for strain $<15 \%$; (c) Stress-strain curves of pPDMD/CNT foams with $25 \mathrm{mg} / \mathrm{cm}^{3}$ of CNTs measured under uniaxial compression (loading and unloading, 20 full cycles) at three different strain rates, namely 0.5 (green line), 5 (blue line), and $10 \mathrm{~mm} / \mathrm{min}$ (red line), and same strain range 0-60\%; (d) Stress-strain curves of pPDMD/CNT foams with $25 \mathrm{mg} / \mathrm{cm}^{3}$ of CNTs measured under uniaxial compression (loading and unloading, 20 full cycles) at three different strain ranges, namely 15 (magenta line), 30 (cyan line), and 60\% (blue line), and same strain rate $10 \mathrm{~mm} / \mathrm{min}$; the inset shows the stress-strain curve of the same foam up to strain of $75 \%$. 

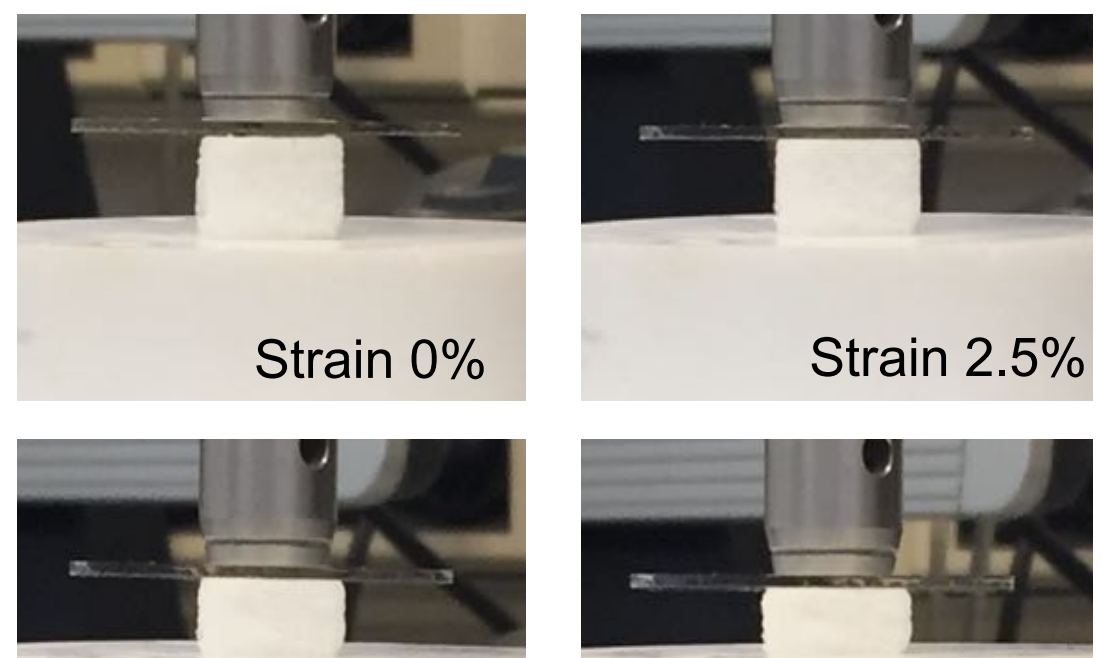

Strain $20 \%$

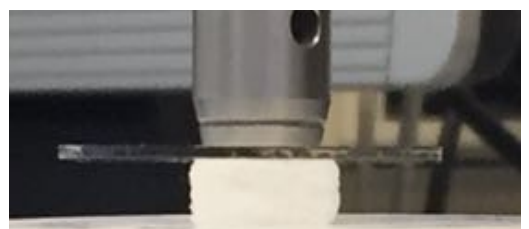

Strain $35 \%$
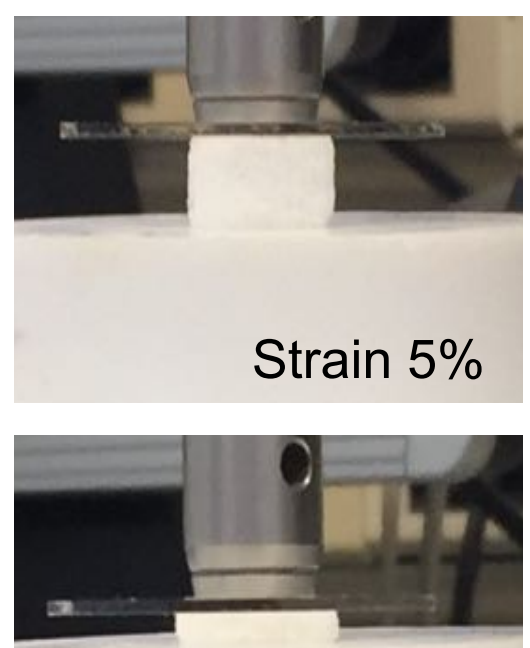

Strain $70 \%$

Figure S3 Evaluation of the Poisson ratio of pPDMS foams with pore sizes of $500 \pm 300 \mu \mathrm{m}$ under uniaxial compressive strain in the range 0-70\%. High-resolution optical pictures of a pPDMS foam with pore sizes of $500 \pm 300 \mu \mathrm{m}$ under different compressive strains from 0 to $70 \%$. It is apparent that there is a limited transverse expansion of the foam under uniaxial longitudinal compression. 

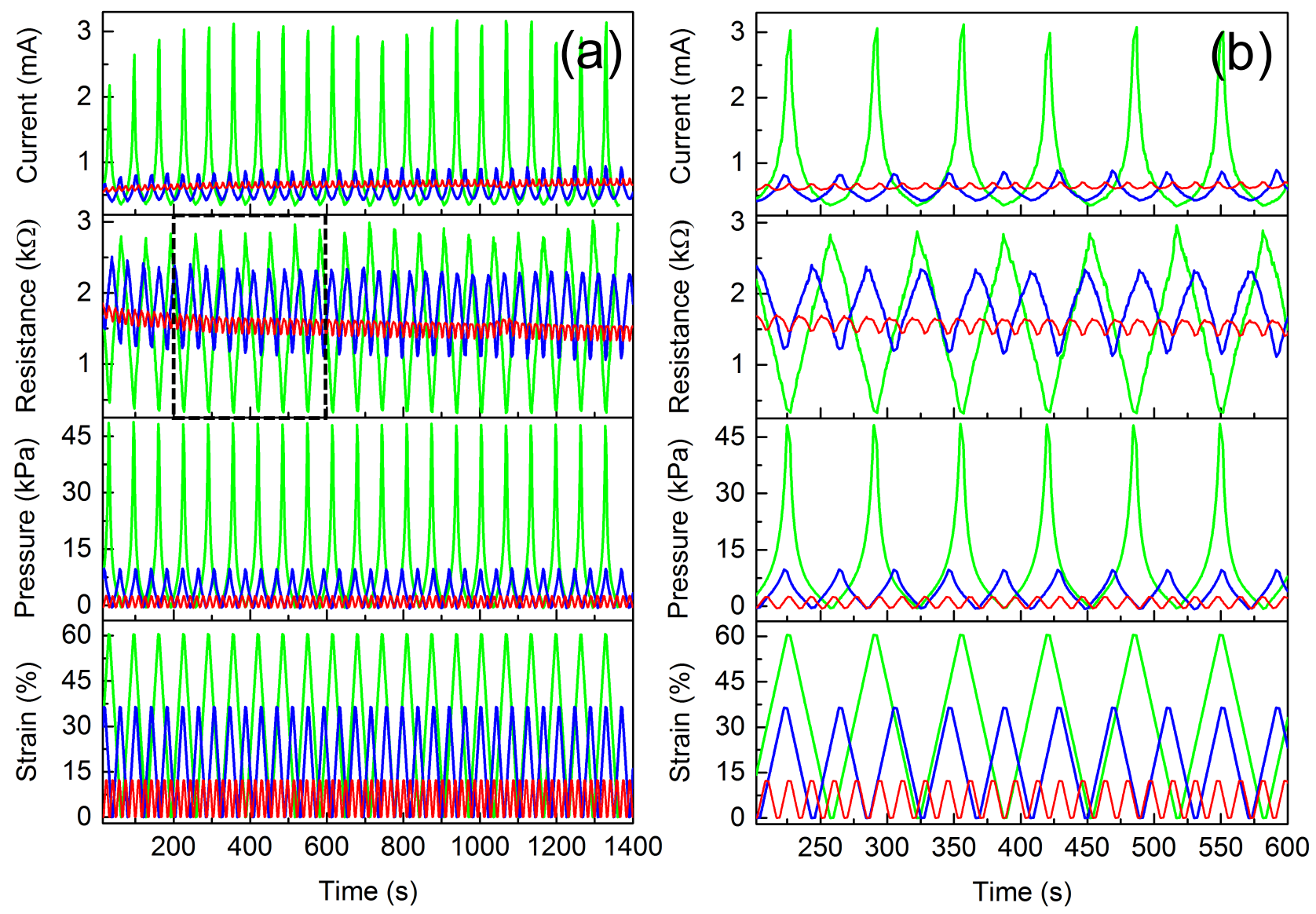

Figure S4 Dynamic piezoresistive characterization of pPDMS/CNT foams decorated with a CNT density of $25 \mathrm{mg} / \mathrm{cm}^{3}$, at $10 \mathrm{~mm} / \mathrm{min}$ and different strain ranges. (a) Time-resolved raw data of current, resistance, and pressure measured over different strain ranges, namely 15 (red line), 30 (blue line), and 60\% (green line) at strain rate of $10 \mathrm{~mm} / \mathrm{min}$. (b) Detail of (a) over a smaller time interval, namely $400 \mathrm{~s}$, highlighting the nice correlation existing between resistance and strain, current and pressure for any strain range tested. 

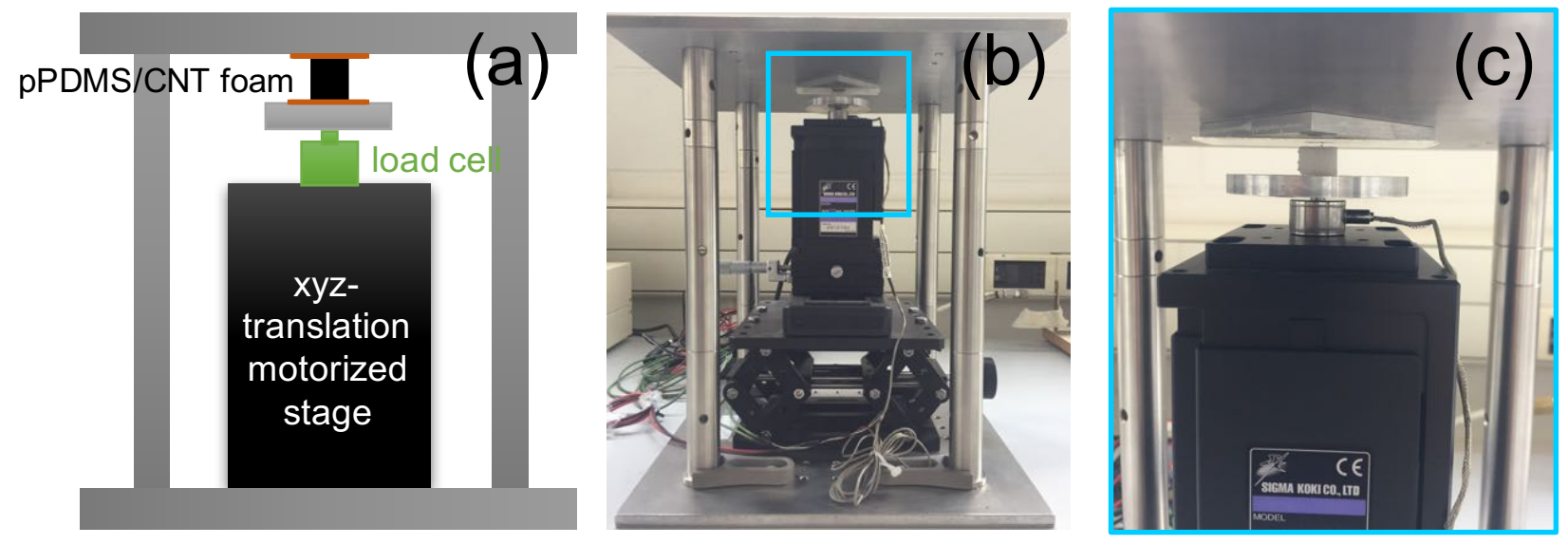

Figure S5 Experimental setup used for the electromechanical characterization of

pPDMS/CNT foams. (a) Schematic and (b, c) photograph of the experimental setup. In (c) a magnification of $(b)$ is reported, from which the translation motorized stage and the load cell can be better appreciated. 

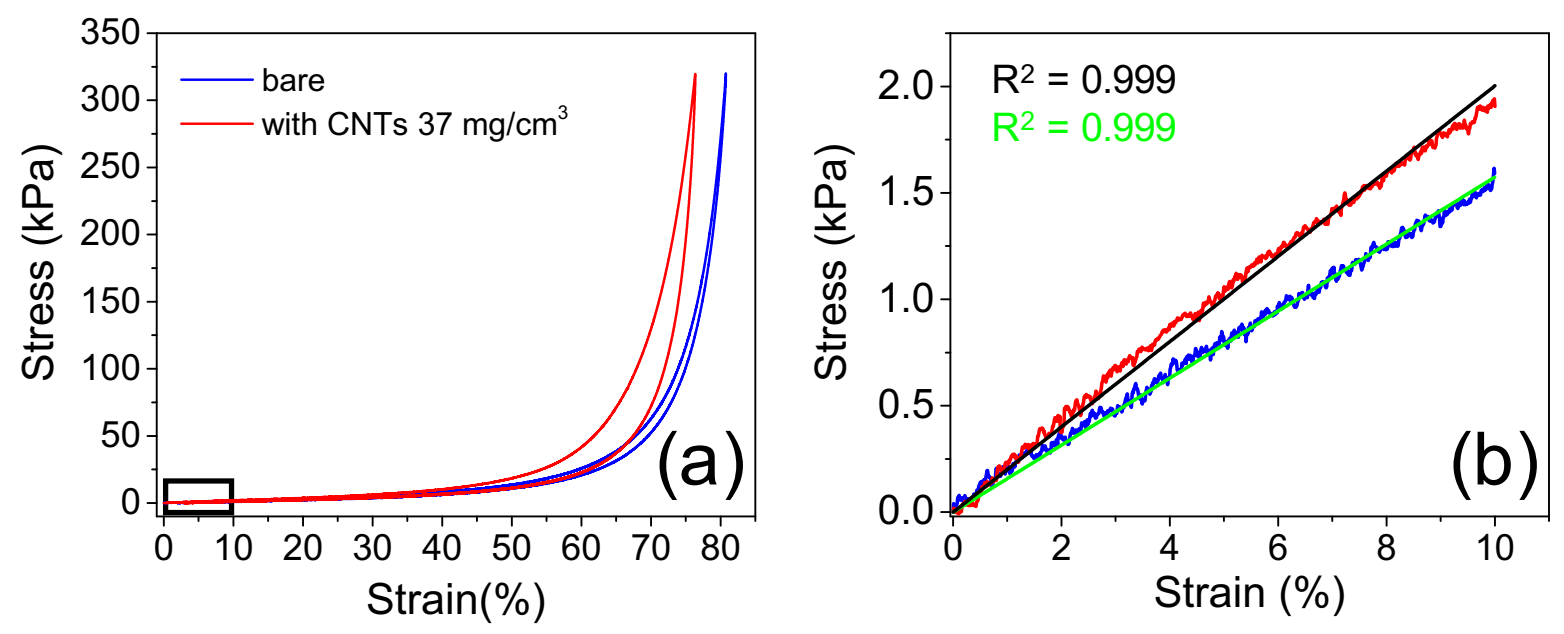

Figure S6 Measurement of the elastic modulus of pPDMS/CNT foams at low strain. (a) Stressstrain curves of pPDMS foams without (blue trace) and with CNTs at density of $37 \mathrm{mg} / \mathrm{cm}^{3}$ (red trace), measured under uniaxial compression (loading/unloading) at strain rate of $0.5 \mathrm{~mm} / \mathrm{min}$. (b) Magnification of the stress-strain curves within the strain range $0-10 \%$, over which the elastic modulus of the foams is calculated. A good linear relationship between stress and strain in this range is apparent, as also pointed out by linear regressions (solid, back and green traces) best fitting experimental data with $\mathrm{R}^{2}=0.999$. 